\title{
Downregulation of GeBP-like a factor by MiR827 suggests their involvement in senescence and phosphate homeostasis
}

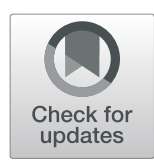

\author{
Chao Ma ${ }^{1,2}$, Qiuju Chen', Shiping Wang ${ }^{1}$ and Amnon Lers ${ }^{2 *}$ (D)
}

\begin{abstract}
Background: Leaf senescence is a genetically controlled degenerative process intimately linked to phosphate homeostasis during plant development and responses to environmental conditions. Senescence is accelerated by phosphate deficiency, with recycling and mobilization of phosphate from senescing leaves serving as a major phosphate source for sink tissues. Previously, miR827 was shown to play a significant role in regulating phosphate homeostasis, and induction of its expression was also observed during Arabidopsis leaf senescence. However, whether shared mechanisms underlie potentially common regulatory roles of miR827 in both processes is not understood. Here, we dissect the regulatory machinery downstream of miR827.

Results: Overexpression or inhibited expression of miR827 led to an acceleration or delay in the progress of senescence, respectively. The transcriptional regulator GLABRA1 enhancer-binding protein (GeBP)-like (GPLa) gene was identified as a possible target of miR827. GPLa expression was elevated in miR827-suppressed lines and reduced in miR827-overexpressing lines. Furthermore, heterologous co-expression of pre-miR827 in tobacco leaves reduced GPLa transcript levels, but this effect was eliminated when pre-miR827 recognition sites in GPLa were mutated. GPLa expression is induced during senescence and its inhibition or overexpression resulted in senescence acceleration and inhibition, accordingly. Furthermore, GPLa expression was induced by phosphate deficiency, and overexpression of GPLa led to reduced expression of phosphate transporter 1 genes, lower leaf phosphate content, and related root morphology. The encoded GPLa protein was localized to the nucleus.

Conclusions: We suggest that MiR827 and the transcription factor GPLa may be functionally involved in senescence and phosphate homeostasis, revealing a potential new role for miR827 and the function of the previously unstudied GPLa. The close interactions between senescence and phosphate homeostasis are further emphasized by the functional involvement of the two regulatory components, miR827 and GPLa, in both processes and the interactions between them.
\end{abstract}

Keywords: Senescence, Arabidopsis thaliana, miRNA, miR827, GPL, Phosphate homeostasis

\footnotetext{
*Correspondence: alers@volcani.agri.gov.il

${ }^{2}$ Department of Postharvest Science, Agricultural Research Organization,

Volcani Center, HaMaccabim Road 68, 7505101 Rishon LeZion, Israel

Full list of author information is available at the end of the article
}

(c) The Author(s). 2021 Open Access This article is licensed under a Creative Commons Attribution 4.0 International License, which permits use, sharing, adaptation, distribution and reproduction in any medium or format, as long as you give appropriate credit to the original author(s) and the source, provide a link to the Creative Commons licence, and indicate if changes were made. The images or other third party material in this article are included in the article's Creative Commons licence, unless indicated otherwise in a credit line to the material. If material is not included in the article's Creative Commons licence and your intended use is not permitted by statutory regulation or exceeds the permitted use, you will need to obtain permission directly from the copyright holder. To view a copy of this licence, visit http://creativecommons.org/licenses/by/4.0/ The Creative Commons Public Domain Dedication waiver (http://creativecommons.org/publicdomain/zero/1.0/) applies to the data made available in this article, unless otherwise stated in a credit line to the data. 


\section{Background}

Leaf senescence is a genetically controlled degenerative process that leads to cell death [1-3]. During senescence, the cellular structure, metabolic activities, and physiological role of the leaf are greatly altered. Chloroplasts degenerate and the photosynthetic apparatus disassembles [4, 5]. Biochemical processes that occur at the onset of senescence include altered gene expression, macromolecule degradation, membrane destabilization, and fluctuations in hormone levels $[3,6]$. It is suggested that during the evolution of senescence, different biochemical, cellular, integrative, and adaptive systems were progressively added to the ancient primary core process as the evolving plant encountered new environmental and developmental contexts [7]. Senescence occurs as part of normal development; however, it can be induced prematurely by stressful environmental stimuli $[8,9]$.

To cope with nutrient-deprived environments, plants must sense changes in external and internal mineral nutrient concentrations and adjust their nutrient metabolisms to meet the demands of plant growth [10]. In annual plants, including Arabidopsis, leaf senescence is highly responsive to nutrient stress. A limited supply of micro- or macronutrients can induce senescence in leaves in order to mobilize resources, such as inorganic phosphate $(\mathrm{Pi})$ and nitrate, to the younger sink organs, thereby ensuring reproduction and contributing to nutrient-use efficiency [11-16]. The leaf is a sink for nitrogen $(\mathrm{N})$ and mineral nutrients during the early stages of development and becomes a nutrient source once it begins to senesce. Senescence involves complex metabolic changes to enable nutrient recycling and allocation [17-19]. Nutrient salvage from older leaves has the adaptive value of recycling nutrients that may be limiting in the environment or that are energetically costly to acquire $[14,17,20]$.

Senescence is characterized by significant changes in gene expression patterns [21, 22], including repression of genes, such as those associated with photosynthesis, and induction of others, including senescence-associated genes (SAGs) that participate in different metabolic and regulatory aspects of senescence [22, 23]. The available knowledge about senescence regulation clearly suggests that it is composed of a complex of pathways forming a network responsible for activation of the different SAGs $[1,24,25]$. At least some of the SAGs' regulation occurs at the transcriptional level [26-28]. The complexity of senescence regulation is also demonstrated by the involvement of a vast number of different transcription factors from different families [24, 29]. The involvement of microRNAs (miRNAs) in senescence regulation has been recently reviewed [30, 31]. In Arabidopsis, miR164 was identified as a key player in senescence through control of its target, the senescence transcriptional activator gene ORE1/AtNAC092 [32]. MiR319 was also shown in Arabidopsis to positively regulate leaf senescence by regulating jasmonic acid biosynthesis [33]. Additional studies suggest the involvement of other miRNAs in senescence [34-36]. We previously conducted large-scale analyses to identify senescence-inducible miRNAs [37]. Many of the identified miRNAs were reported to be involved in nutrient responsiveness, which is consistent with the nutrient-remobilization process taking place during senescence. Among these, miR827 was found to be significantly induced during leaf senescence, its expression peaking in late senescence [37]. MiR827 was shown to play significant roles in regulating $\mathrm{Pi}$ homeostasis in plants in a nitrate-dependent fashion [38-41]. Pi deprivation results in induction of miR827 expression, which post-transcriptionally represses transcript accumulation of its target gene nitrogen limitation adaptation (NLA) [39, 42]. NLA can mediate the ubiquitination and degradation of the plasma-membrane-localized phosphate transporter 1 (PHT1) family of $\mathrm{Pi}$ transporters, thereby affecting Pi homeostasis [39].

Maintenance of $\mathrm{Pi}$ homeostasis is crucial for crop production [43, 44]. Low levels of available Pi in the soil limit biomass and yield potential, and therefore, remobilization of $\mathrm{Pi}$ within the plant is important $[44,45]$. Pi remobilization from senescing leaves is a major source of Pi for sink tissues. While it is clear that plants are efficient at recycling Pi from senescing leaves, our knowledge of the molecular components involved in the process is limited compared to studies of $\mathrm{N}$ remobilization [46, 47]. Plants can remobilize over $50 \%$ of the Pi from senescing leaves [48]. Thus, translocation of Pi from older to developing leaves is quantitatively important. Pi remobilization and transportation are effected by the action of hormones, transcription factors, and Pi-scavenging enzymes [47]. Pi transporters are critical for Pi allocation and remobilization within plants [49-51]. The Arabidopsis Pi transporter PHT1;5 mobilizes Pi between source and sink organs and influences the interaction between $\mathrm{Pi}$ homeostasis and ethylene signaling [50]. PHT1;5 overexpression resulted in altered $\mathrm{Pi}$ remobilization and premature senescence. PHT4;6 was also found to be involved in senescenceassociated processes in Arabidopsis [52].

In this report, the close interactions between senescence and $\mathrm{Pi}$ homeostasis are further emphasized by the functional involvement of two regulatory components: miR827 and the GLABRA1 enhancer-binding protein $(G e B P)$-like regulatory factor GPL $\alpha$, in both processes.

\section{Results}

Altered expression of miR827 is associated with modified leaf senescence

Our previous analysis revealed that mature miR827 is highly induced during leaf senescence [37]. Expression 
analyses of pre-miR827 resulted in similar kinetics and fold induction, thus verifying enhanced transcript accumulation during early and late stages of natural senescence, as well as during dark-induced senescence of detached leaves (Additional file 1: Figure S1A, C). To determine whether senescence induction by miR827 is transcriptionally regulated, a ca. $1.3-\mathrm{kb}$ sequence upstream of the transcription start site was cloned in front of the GFP reporter gene to construct miR827Pro:GFP. Arabidopsis plants transformed with this vector were examined for GFP expression. In both natural and artificial senescence of detached leaves, GFP expression was induced during late senescence, with the GFP signal colocalizing with yellowing sections of the leaves (Additional file 1: Figure S1B, D).

To investigate the possible involvement of miR827 in senescence, pre-miR827 expression was modified. For overexpression, the $35 \mathrm{~S}$ constitutive promoter was cloned in front of the precursor sequences of miR827 and the constructed vector (Additional file 2: Figure $\mathrm{S} 2 \mathrm{~A})$ was used to generate transgenic miR827- overexpressing plants in which pre-miR827 transcript was strongly induced, about 300-fold compared to the wild type (Additional file 2: Figure S2B). Suppression of miR827 was achieved by employing the target mimicry method (short tandem target mimic-STTM) (FrancoZorrilla et al., 2007; Yan et al., 2012). The miR827STTM sequence was overexpressed by the $S 35$ promoter (Additional file 2: Figure S2A), and in the resulting Arabidopsis plants, miR827 transcript level was less than $10 \%$ of that measured in the wild type (Additional file 2: Figure S2B). In plants overexpressing pre-miR827, accelerated natural senescence of attached leaves was observed as well as acceleration of dark-induced senescence (Fig. 1a, d). Early senescence in the miR827overexpressing lines was indicated by an accelerated decline in chlorophyll and protein contents in sameposition leaves (Fig. 1b, c, e, f). In plants suppressed of miR827 expression retardation of senescence was observed, as indicated by late development of yellowing in the miR827-STTM lines (Fig. 1a, d), as well as by a retarded decrease in chlorophyll and protein contents in
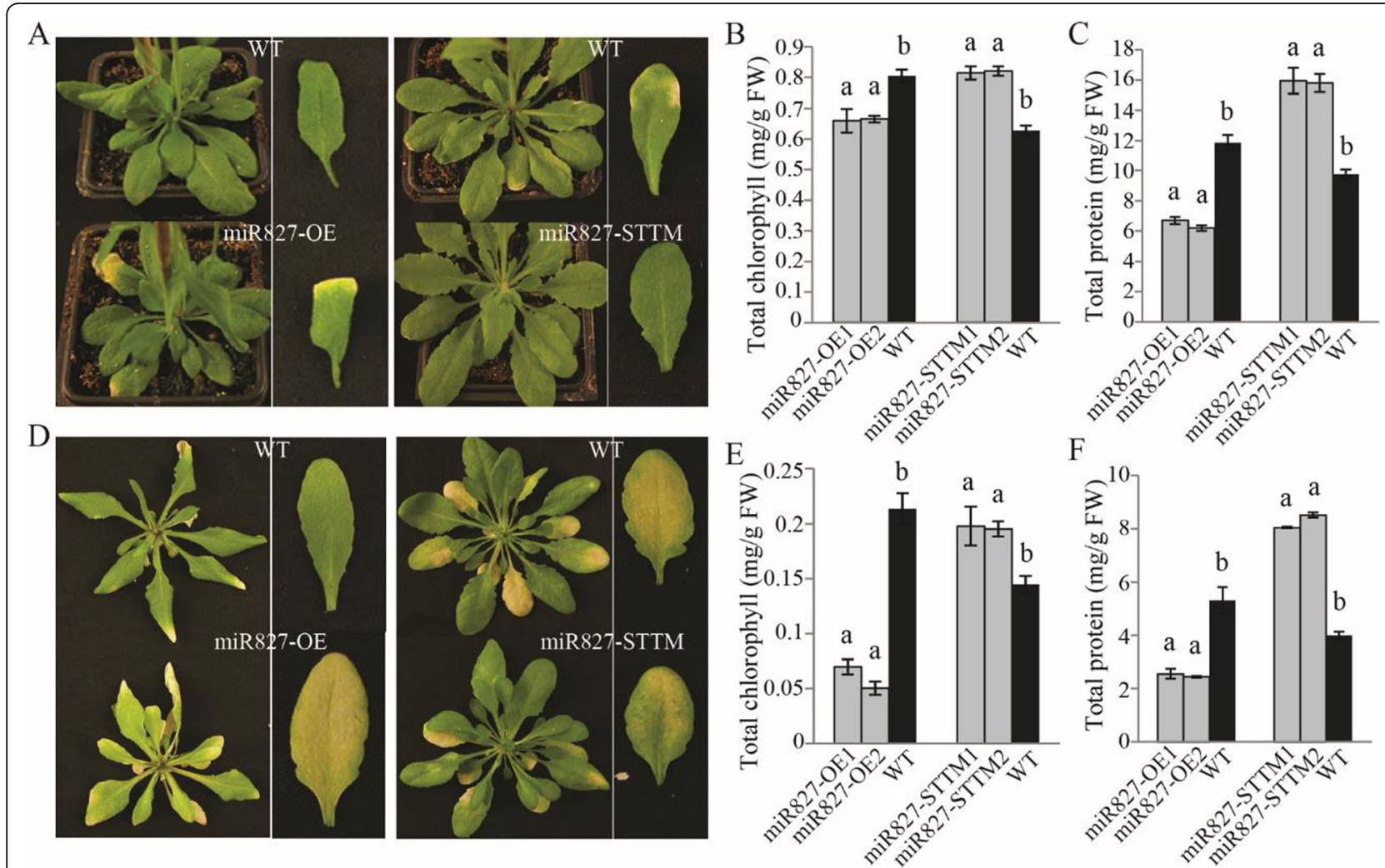

Fig. 1 Altered expression of miR827 in transgenic plants results in modified progression of leaf senescence. $\mathbf{a}, \mathbf{d}$ Enhanced senescence induces leaf yellowing in transgenic plants overexpressing miR827 (miR827-OE) during natural leaf senescence (a, left panel) and during artificial darkinduced senescence (d, left panel). Retardation of senescence-induced yellowing in miR827-silenced transgenic plants (miR827-STTM) during natural leaf senescence (a, right panel) and during artificial dark-induced senescence (d, right panel). b, c Effects of overexpression or suppression of miR827 on total chlorophyll (b) and protein (c) contents during natural leaf senescence. $\mathbf{e}, \mathbf{f}$ Effects of overexpression or suppression of miR827 on total chlorophyll (e) and protein (f) contents during dark-induced leaf senescence. Three independent transgenic lines were examined. Different letters above the columns indicate significant differences within each compared triplet $(P<0.05$, Student's $t$ test, \pm SD). WT, wild type 
those lines compared to the wild type during both natural and dark-induced senescence (Fig. 1b, c, e, f).

\section{Consequences of altered miR827 expression to gene expression}

NLA (At1G02860) has been validated as a target of miR827 in Arabidopsis [42, 53], and PHT5;1/VPT1 (At1G63010) is a suggested target of miR827 [42]. PHT5;1/VPT1 is essential for Pi homeostasis [54, 55]. A search for additional candidate miR827 targets using psRNATarget [56] suggested a GPL gene (At4G00610), hereafter termed GPL $\alpha$.

The predicted miR827-recognition site in the GPL $\alpha$ sequence is shown in Additional file 3: Figure S3. To examine actual cleavage of GPL $\alpha, 5^{\prime}$ RACE (rapid amplification of cDNA ends) assay was employed. Cleavage of GPL $\alpha$ in Arabidopsis could not be identified within the predicted target site; it was, however, identified (in 5 out of 13 incidences examined) 10 bases downstream of the 3 '-end of the putative recognition site and an additional 8 cleavage sites were spread further downstream (Additional file 3: Figure S3). To further examine the relationship between $m i R 827$ and GPL $\alpha$, we transiently co-expressed both in tobacco leaves using Agrobacterium infiltration assay and examined the consequences to GPL $\alpha$ transcript level. Coexpression of GPL $\alpha$ with pre-miR827 resulted in a ca. 3fold decrease in its transcript level compared to coexpression with the empty cloning vector as a control (Fig. 2a). Furthermore, mutations in the predicted miR827-recognition site in GPL $\alpha$ (Additional file 3: Figure S3) resulted in nullification of the observed miR827- mediated decrease in GPL $\alpha$ transcript level (Fig. 2a), supporting functional involvement of miR827 in the regulation of GPL $\alpha$. These mutations by themselves did not affect GPL $\alpha$ stability (Fig. 2a). Control experiments revealed the inability of grape $V v-m i R 171$, which is unrelated to $G P L \alpha$, to affect its transcript levels, whereas it was functional in reducing the transcript level of its verified target $V v$-SCL15 (Fig. 2b, c [57]).

Experiments were performed to examine the effects of altered miR827 expression on its validated target gene $N L A$, as well as on PHT5;1 and GPL $\alpha$ expression. Expression of NLA, PHT5;1, and GPL $\alpha$ was measured during young, mature, early senescence and late senescence stages [37]. In the wild type, all genes were induced toward the late stage of senescence (Fig. $3 \mathrm{a}-\mathrm{c}$ ). NLA expression was low at the young, mature, and early senescence stages and then increased in late senescence, as previously reported (Fig. 3a) [37]. PHT5;1 expression declined at the mature leaf stage compared to that measured at the young leaf stage and then increased again during early and late senescence stages (Fig. 3b). Expression of GPL $\alpha$ increased only in late senescence (Fig. 3c). To determine the effects of altering miR827 expression on these three genes, their expression was measured in transgenic miR827-overexpressing and silenced plants. A clear increase in the transcript levels of NLA, PHT5;1, and GPL $\alpha$ was observed in the two miR827-STTM lines, whereas their transcript levels were reduced in the two miR827-overexpressing lines (Fig. 3a-c).

To further examine the relationship between miR827 and GPL $\alpha$ expression, the latter was characterized in

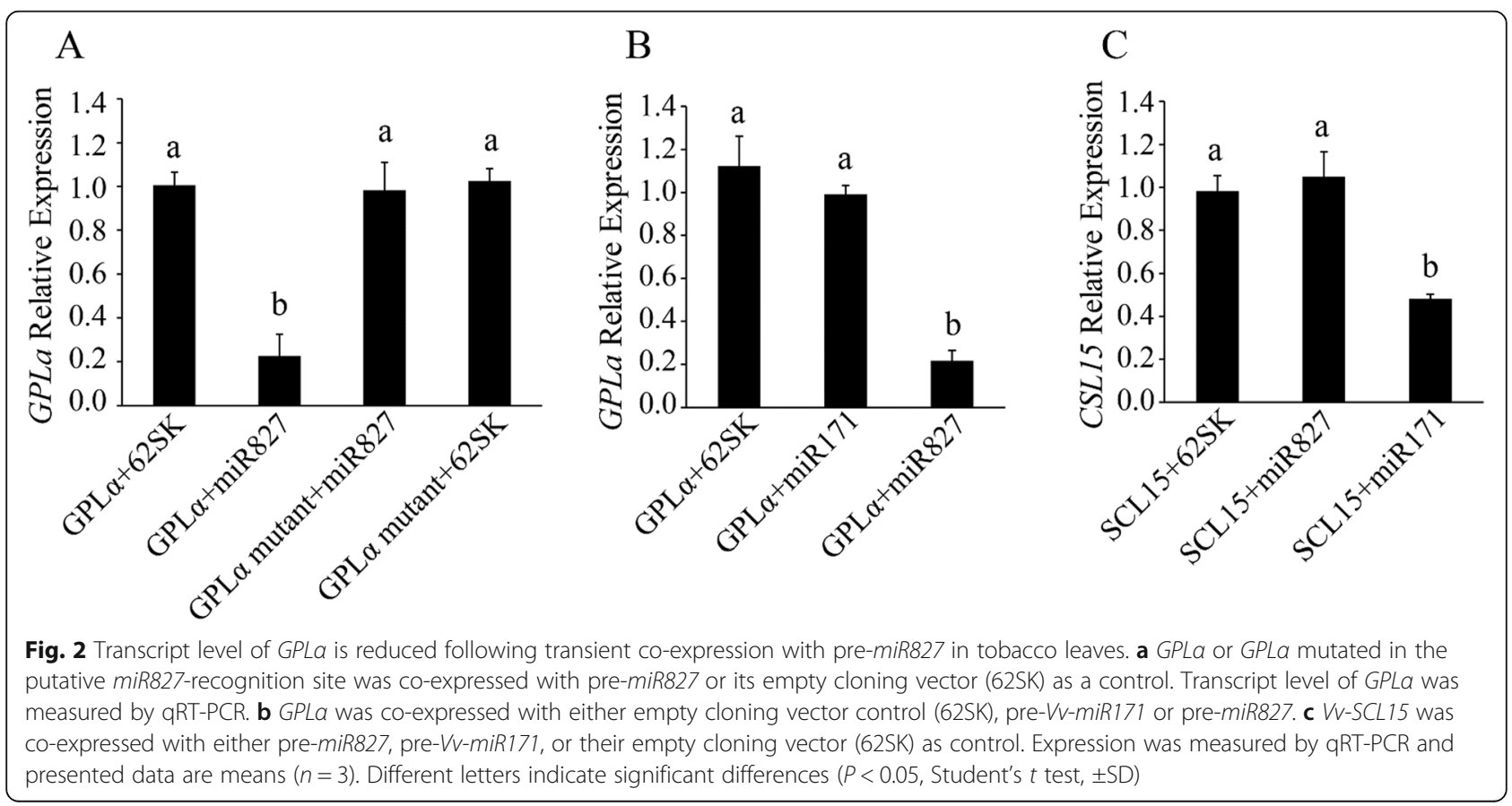




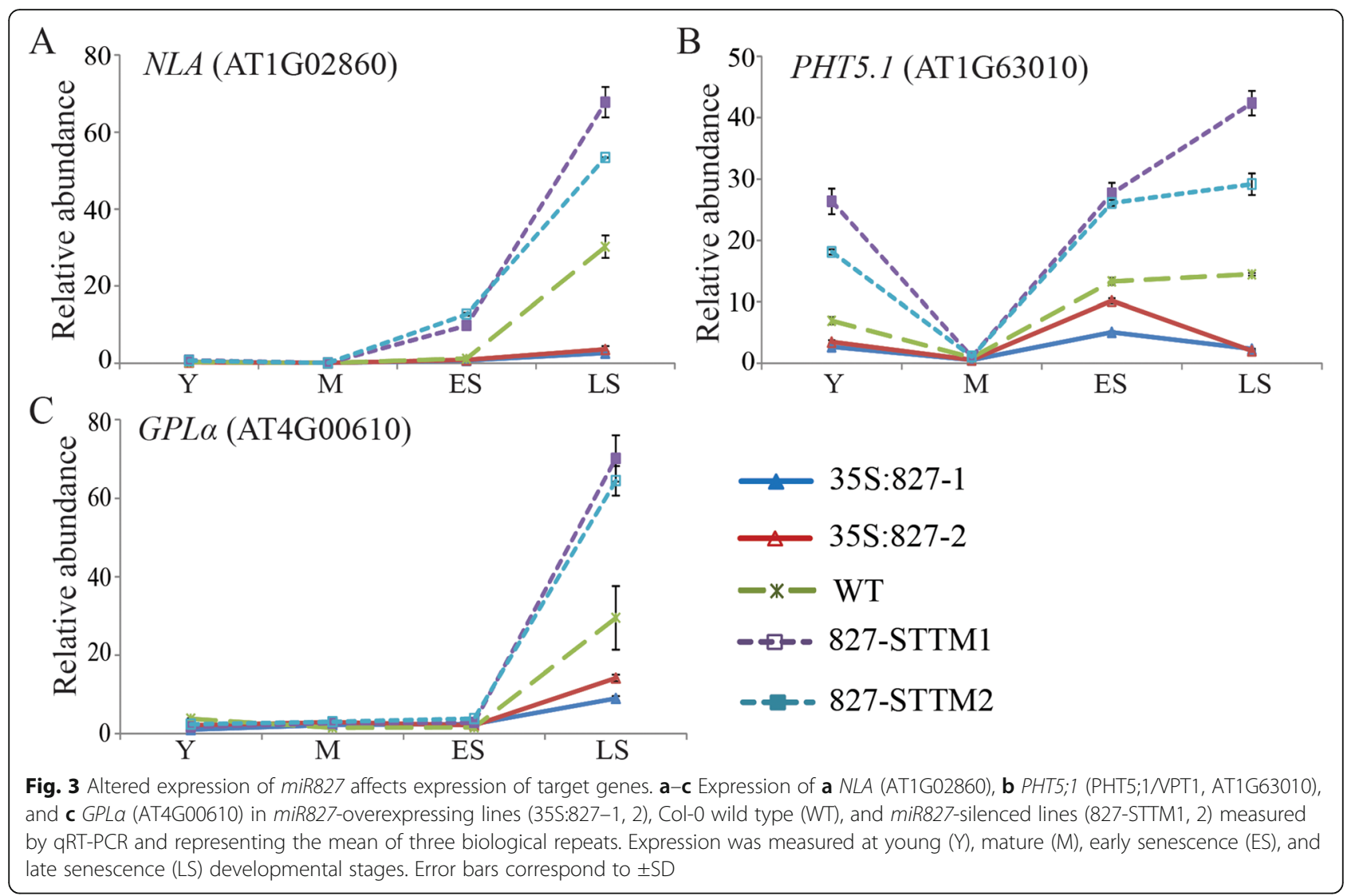

miR827-overexpressing and miR827-silenced transgenic lines and compared to the expression of two SAGsBFN1 (a senescence-induced nuclease [58]) and SAG12 (a senescence-induced protease [59]) - as markers of senescence progression. The expression of GPL $\alpha, B F N 1$, and $S A G 12$ was measured at different times during natural leaf senescence and plotted versus chlorophyll content, to enable comparing their expression in the miR827-altered lines. Expression of the genes in the wild type was generally correlated and showed an accelerated increase when chlorophyll level was reduced to about $0.5 \mathrm{mg} / \mathrm{g}$ fresh weight (Additional file 4: Figure S4). Expression of the three genes was then examined in the transgenic miR827-overexpressing Arabidopsis in which senescence is enhanced. Expression of GPL $\alpha$ was suppressed whereas expression of both BFN1 and SAG12 continued to be induced as senescence progressed and chlorophyll levels decreased, suggesting an effect of miR827 on GPLa expression (Additional file 4: Figure S4). On the other hand, expression of GPL $\alpha$ in the miR827-STTM lines was considerably higher than that of BFN1 and SAG12 (Additional file 4: Figure S4), whereas in the wild type, it was lower. The expression of $B F N 1$ and SAG12 was induced at a later stage of senescence in the miR827-STTM lines, as indicated by a rise in their transcript level when chlorophyll content decreased, relative to that observed in the wild type (Additional file 4: Figure S4).

\section{Altered GPLa expression affects senescence progression}

GPL $\alpha$ expression increased significantly, mainly during the late stage of natural and dark-induced senescence (Additional file 5: Figure S5A, C). To determine whether senescence induction of GPL $\alpha$ is regulated by its promoter, sequences encompassing about $1.3 \mathrm{~kb}$ upstream of the transcription start site were cloned in front of the GFP coding sequence to construct GPL $\alpha$ Pro:GFP. Transgenic Arabidopsis plants transformed with this vector were examined for GFP expression by following fluorescence. In both natural and artificially (dark)-induced senescence, GFP expression was induced during advanced senescence stages and the GFP signal overlapped with yellowing leaf sections (Additional file 5: Figure S5B, D).

To investigate the involvement of GPL $\alpha$ in senescence, its expression was either suppressed or overexpressed in the two Arabidopsis accessions: Columbia (Col-0) and Landsberg erecta (Ler). Vectors for generating GPL $\alpha$ overexpressing lines were constructed by activating GPL $\alpha$ with the $35 S$ constitutive promoter (Additional file 6: Figure S6A), resulting in GPL $\alpha-\mathrm{OE} 1$ and GPL $\alpha-\mathrm{OE} 2$ (Col-0), and GPL $\alpha-\mathrm{OE} 3$ and GPL $\alpha-\mathrm{OE} 4$ 
(Ler). GPL $\alpha$ expression in the transgenic lines was about 100-fold higher than in the wild type (Additional file 6: Figure S6B, C). Suppression of GPL $\alpha$ expression using an RNA interference (RNAi) construct (Additional file 6: Figure S6A) resulted in GPL $\alpha-S I 1$ and GPL $\alpha-S I 2$ (Col0 ), and GPL $\alpha-\mathrm{SI} 3$ and GPL $\alpha-\mathrm{SI} 4$ (Ler), in which GPL $\alpha$ expression was suppressed to less than $10 \%$ of that measured in the wild type (Additional file 6: Figure S6B, C). In addition, two Arabidopsis T-DNA insertional mutants in GPLa were used: ET2099.Ds3.07.28.00.b.544 (Col-0 background) termed GPL $\alpha-\mathrm{KO} 1$ and GK-252G05014573 (Ler background) termed GPL $\alpha-\mathrm{KO} 2$ (Additional file 6: Figure S6A). In both of these mutants, expression of GPL $\alpha$ was suppressed to less than $10 \%$ of that measured in the respective wild types (Additional file 6: Figure S6D).

$G P L \alpha$ overexpression resulted in inhibition of both natural (Fig. 4a) and dark-induced (Fig. 4d) senescence in the two independent lines compared to the wild type (Fig. 4a, d). Delayed senescence in the GPL $\alpha$-overexpressing lines was indicated by a retarded decline in both chlorophyll and protein contents in same-position leaves compared to the wild types during natural senescence (Fig. 4b, c) and dark-induced senescence (Fig. 4e, f). Similar retardation of senescence was observed in the Ler accession (Additional file 7: Figure S7). Suppression of GPL $\alpha$ expression resulted in acceleration of both natural (Additional file 7: Figure S7A) and darkinduced (Additional file 7: Figure S7D) senescence. Accordingly, measurements of chlorophyll and protein contents in the GPL $\alpha$-suppressed lines revealed lower levels than those in the wild type during both natural (Additional file 7: Figure S7B, C) and dark-induced (Additional file 7: Figure S7E, F) senescence. Accelerated senescence was apparent when suppression of GPL $\alpha$ expression was achieved by either RNAi or T-DNA mutations (Fig. 4). Similar results were obtained for the Ler accession (Additional file 7: Figure S7).

Regulation of miR827 and GPLa expression in response to Pi availability and nuclear localization of GPLa

MiR827 shows increased expression in response to reduced Pi availability [38, 42]. To learn more about the responses of both GPL $\alpha$ and $m i R 827$ to Pi limitation, their expression was measured simultaneously in response to transfer from full-nutrient medium to a $\mathrm{Pi}$ -

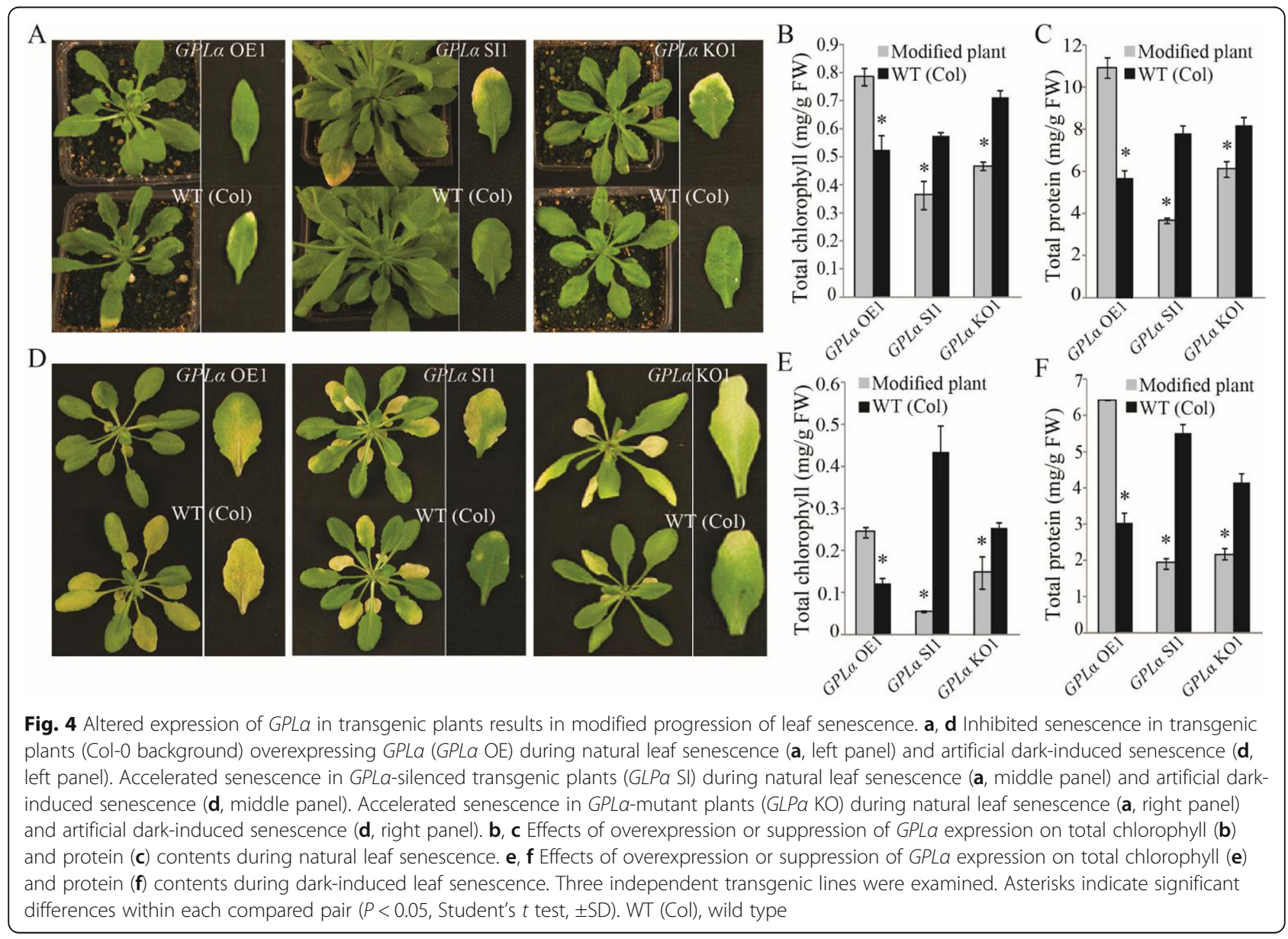


deficient one. Arabidopsis seedlings (Col-0 and Ler) were grown for 10 days on half-strength MS medium and then transferred to either full-nutrient or Pi-deficient medium for 10 days and both miR827 and GPL $\alpha$ transcript levels were measured by quantitative (q) RT-PCR. Expression of miR827, in both accessions, was found to be induced about 10- to 15-fold under Pi deficiency compared to that measured in the full-nutrient medium, as reported previously [38] (Fig. 5A). GPL $\alpha$ expression was also induced, albeit more moderately, under Pi deficiency, increasing to about 4- and 7-fold compared to growth on full-nutrient medium in Col-0 and Ler accessions, respectively (Fig. 5B).

Expression kinetics of miR827 and GPL $\alpha$ was simultaneously measured following transfer of 10-day-old seedlings from full-nutrient to Pi-deficient medium. Leaf and root tissues were sampled daily for 11 days following the transfer. In leaves, miR827 expression responded rapidly, in a matter of hours, to the change: it began to increase linearly, reaching about 150 -fold its initial expression on day 2 (Additional file 8: Figure S8A). After this striking increase, expression decreased until day 7 to a level that was about 20 -fold higher than its initial one; after that, it remained fairly constant (Additional file 8: Figure S8A). Expression of GPL $\alpha$, measured simultaneously in the leaves, changed very mildly, with a tendency to increase until day 4 when it was sharply induced and increased about 25-fold compared to its initial expression (Additional file 8: Figure S8A). After this peak, GPL $\alpha$ expression decreased close to its initial expression level.

In the roots, miR827 expression was induced following exposure to Pi deficiency, similar to its kinetics in the leaves, but to a lower level (Additional file 8: Figure S8B). MiR827 expression peaked after 2 days of $\mathrm{Pi}$ deficiency, to about 8 -fold its initial level, followed by a decrease toward day 7 , after which it remained low. Expression of GPL $\alpha$ remained low initially, increasing only slightly during the first 2 days after exposure to Pi deficiency; this was followed by an increase in expression level to a peak on day 4, at about 12-fold its initial expression (Additional file 8: Figure S8B). This increase occurred concomitantly with the reduction in miR827 expression level.

To determine whether induction of miR827 and GPL $\alpha$ is regulated by the genes' upstream promoters, about $1.3 \mathrm{~kb}$ upstream of the transcription start site were cloned for each of the genes in front of the GFP coding sequence to construct vectors miR827Pro:GFP and GPL $\alpha$ Pro:GFP. Transgenic plants transformed with these vectors were examined for GFP expression by fluorescence imaging. For both promoters, Pi deficiency resulted in induced GFP signal in both leaves and roots
(Fig. 5C, D). Under full Pi sufficiency, no GFP signal was detected (Fig. 5C, D).

To localize GPL $\alpha$ protein in the cells, its coding sequence was translationally fused $3^{\prime}$ to the GFP coding sequence under the control of the $35 S$ promoter and the resulting construct was used in an Arabidopsis transientexpression assay. Confocal microscopy analyses of the leaves revealed localization of the GFP signal to the nuclei due to the presence of the GPL $\alpha$ sequence (Fig. 5F). Localization of GPL $\alpha$-GFP to the nuclei was verified in transient-expression assay in which GFP signal was observed to overlap with DAPI-stained nuclei (Additional file 9: Figure S9). In control plants, which were infiltrated with GFP under the control of the $35 S$ promoter, GFP signal was present in the cytoplasm as well as in the nuclei, as is characteristic for this protein (Fig. 5E). Nuclear localization of GPL $\alpha$ and the presence of putative nuclear localization sequences were also predicted by the bioinformatics tools ePlant [60] and AtSubP [61], accordingly.

\section{MiR827 and GPLa are involved in root response to $\mathrm{Pi}$ availability}

Plant developmental responses to Pi deficiency include major alterations in root architecture, such as a reduction in primary-root growth $[62,63]$. To determine the possible involvement of miR827 and GPL $\alpha$ in this response, we examined root phenotypes in Arabidopsis plants in which expression of these genes was altered. When grown under optimal nutrient conditions, there was no apparent difference in root development between the wild type and either miR827-overexpressing/suppressed or GPL $\alpha$-overexpressing/suppressed plants. Arabidopsis (Col-0) seedlings were grown on Pi-sufficient medium for 10 days and then transferred to Pi-deficient medium for an additional 7-10 days of growth. Plants overexpressing miR827 exhibited accelerated growth rate of the primary root, which was thus about $50 \%$ longer than that in the wild type (Fig. 6a, b). In miR827-suppressed plants grown under Pi deficiency, primary-root elongation was inhibited by about 20\% (Fig. 6a, b). In the Arabidopsis mutants with modified GPL $\alpha$ expression, the opposite effect was observed: under Pi-deficient conditions, primary-root growth was inhibited in the GPL $\alpha$ overexpressing lines, resulting in a low but significant $10 \%$ reduction in average primary-root length (Fig. 6c, d). On the other hand, in transgenic plants in which GPL $\alpha$ expression was suppressed by either RNAi or TDNA insertional mutations, primary-root growth was accelerated under Pi deficiency, resulting in an average $20 \%$ increase in length (Fig. 6c, d). Similar effects of altered GPL $\alpha$ expression on primary-root elongation were observed for the Ler accession (Fig. 6e, f). The acceleration effect of GPL $\alpha$ suppression on root elongation 
A

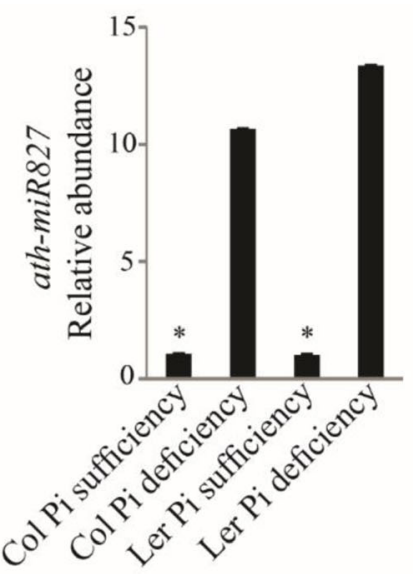

C

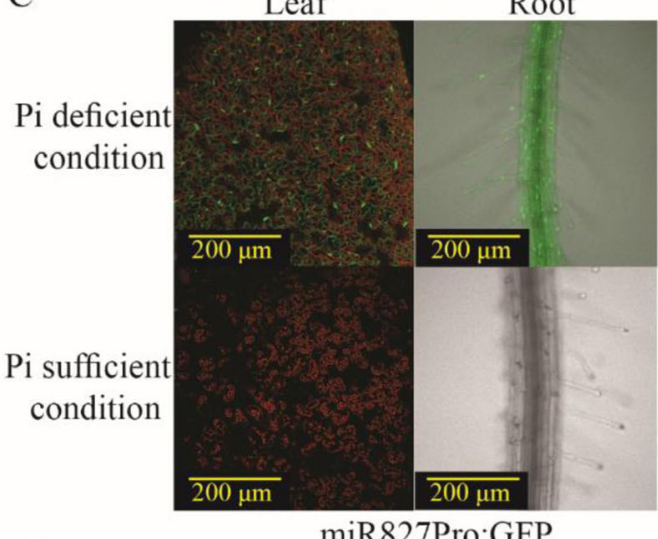

E

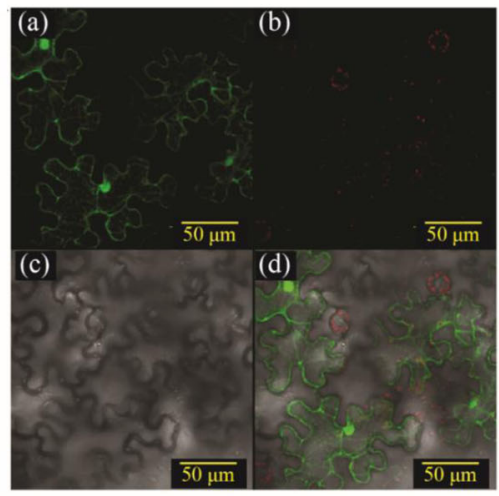

35S:GFP

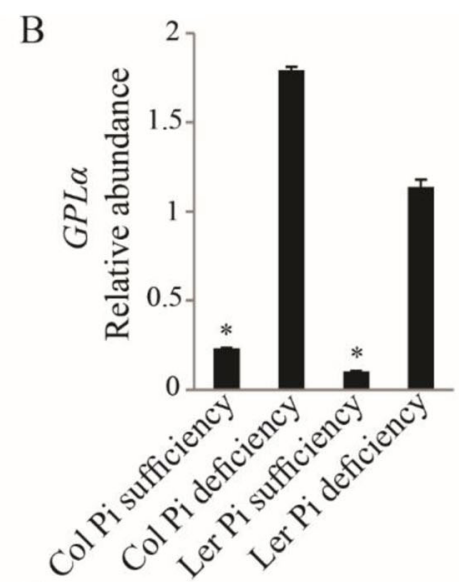

D

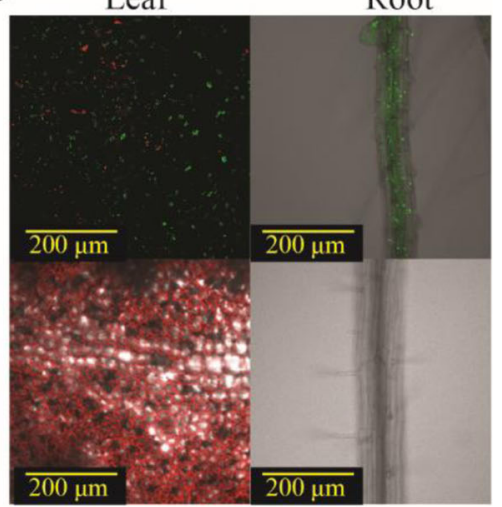

F

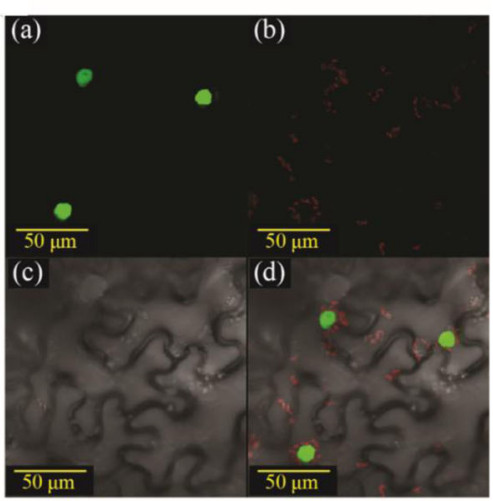

35S:GFP::GPL $\alpha$

Fig. 5 MiR827 and GPLa expression is induced following exposure to Pi-deficient growth conditions and GPLa is localized to Arabidopsis cell nuclei. A and B Measurement of miR827 (A) and GPLa (B) expression in either Col-0 (Col) or Ler wild type 10-day-old seedlings following an additional 10 days under Pi-deficient growth conditions. Asterisks indicate significant differences $(P<0.05$, Student's $t$ test, \pm SD). C and $\mathbf{D}$ GFP fluorescence imaging in leaves and roots of transgenic plants (Col-0 background) containing miR827-promoter-driven GFP (miR827Pro:GFP) (C) or GPLa-promoter-driven GFP (GPLa Pro:GFP) (D) under Pi deficiency (upper panels) or optimal Pi (lower panels). Six independent transgenic lines were examined. Representative lines are shown. $\mathbf{E}$ and $\mathbf{F}$ Arabidopsis wild type (Col-0) leaves were transiently transformed with a vector including the control GFP construct, 35S:GFP (E) or a translational fusion between GPLa and GFP, 35S:GFP::GPLa (F). GFP protein localization was visualized using confocal microscopy. (a) GFP fluorescence; (b) chlorophyll autofluorescence; (c) bright-field image; (d) overlay of $\mathbf{a}$, b, and c

could be reversed by overexpressing GPL $\alpha$ in the GPL $\alpha-$ $\mathrm{KO} 2$ mutant, which resulted in primary-root elongation similar to that measured for the wild type grown under Pi deficiency (Additional file 10: Figure S10).
GPLa is involved in regulation of PHT1 expression in response to $\mathrm{Pi}$ availability

MiR827 is known to be involved in $\mathrm{Pi}$ homeostasis through the transcript-level regulation of its target gene 


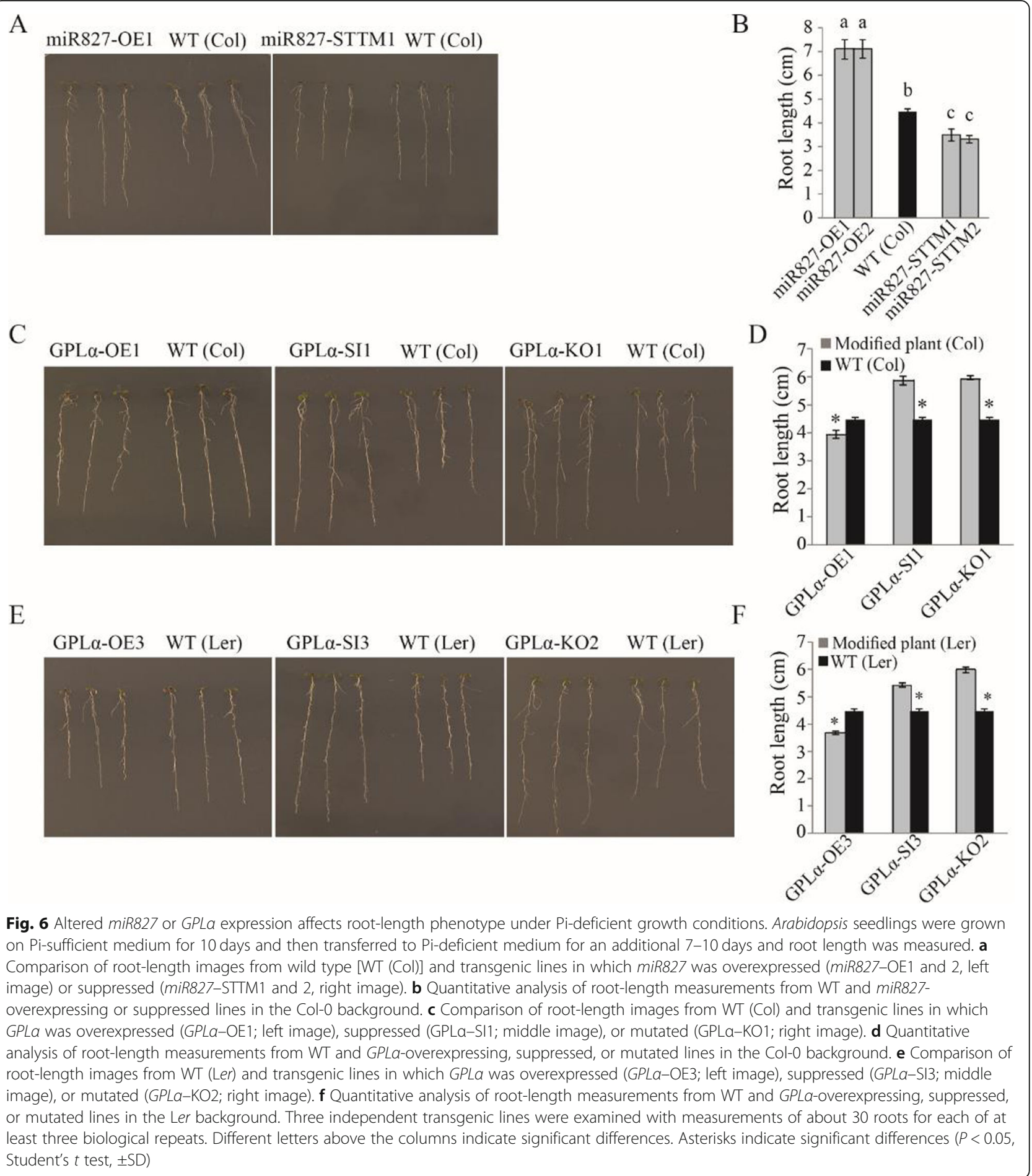

NLA which mediates degradation of the PHT1 family of Pi transporters $[39,64]$. We examined the possible involvement of GPL $\alpha$ in the regulation of PHT1 genes as well. Arabidopsis seedlings grown for 2 weeks on plates with Pi-sufficient medium were transferred to $\mathrm{Pi}$ deficient medium for 7 days, and gene expression was analyzed. To verify that under our working conditions,
Pi-deficiency-induced genes are activated, expression of PHT1 [51] and Phosphate1 (PHO1) [65], encoding for $\mathrm{H}^{+} / \mathrm{Pi}$ cotransporter and the $\mathrm{Pi}$ exporter, respectively, was measured following growth on the Pi-deficient medium. Both PHT1 and PHO1 are known to be regulated indirectly, at least in part, by miR827 and miR399, respectively [40]. Clear and high induction of transcript 
levels of both genes was observed (Additional file 11: Figure S11).

Expression of all nine members of the PHT1 gene family of Arabidopsis [66] was measured in transgenic lines overexpressing GPL $\alpha$. All nine genes had reduced transcript levels in the two independent GPL $\alpha$-overexpressing lines compared to those measured in the wild type following 7 days of growth in Pi-deficient medium (Fig. 7a). For PHT1;1, PHT1;5, and PHT1;7, the reduction was mild, to about $10-30 \%$ of that measured in the wild type (Fig. 7a); for PHT1;3 and PHT1;6, the reduction was very strong, 90 and $65 \%$ of that found in the wild type, respectively (Fig. 7a). For four of the PHT1 genes, PHT1;1 to PHT1;4, similar analyses were performed in transgenic Arabidopsis lines of both Col-0 and Ler accessions in which GPL $\alpha$ was either overexpressed or suppressed. In both accessions and for all four PHT1 genes, expression was reduced in the GPL $\alpha$ overexpressing lines and elevated in the GPL $\alpha$-suppressed lines (Fig. 7b). Whereas the effect was mild for PHT1;1 (Fig. 7b), in PHT1;3, both reduction and induction of expression in the GPL $\alpha$-overexpressed and suppressed lines, respectively, were high (Fig. 7b).

To examine whether GPL $\alpha$ is involved in the regulation of other genes known to be induced by Pi deficiency, their expression was examined in the GPL $\alpha$ overexpressing Arabidopsis lines. No difference in expression was observed between GPL $\alpha$-overexpressing and wild type lines following exposure to Pi deficiency for any of those genes (Additional file 12: Figure S12).

\section{Altered GPLa expression results in modified Pi levels}

GPL $\alpha$ expression was found to be regulated by $\mathrm{Pi}$ deficiency, and expression of PHT1 genes encoding phosphate transporters was altered in transgenic Arabidopsis in which GPL $\alpha$ expression was modified. Experiments were therefore performed to examine the effects of modified GPL $\alpha$ expression on total phosphorus (P) and $\mathrm{Pi}$ content. Modulation of miR827 expression was previously found to affect cellular Pi content, with overexpression of miR827

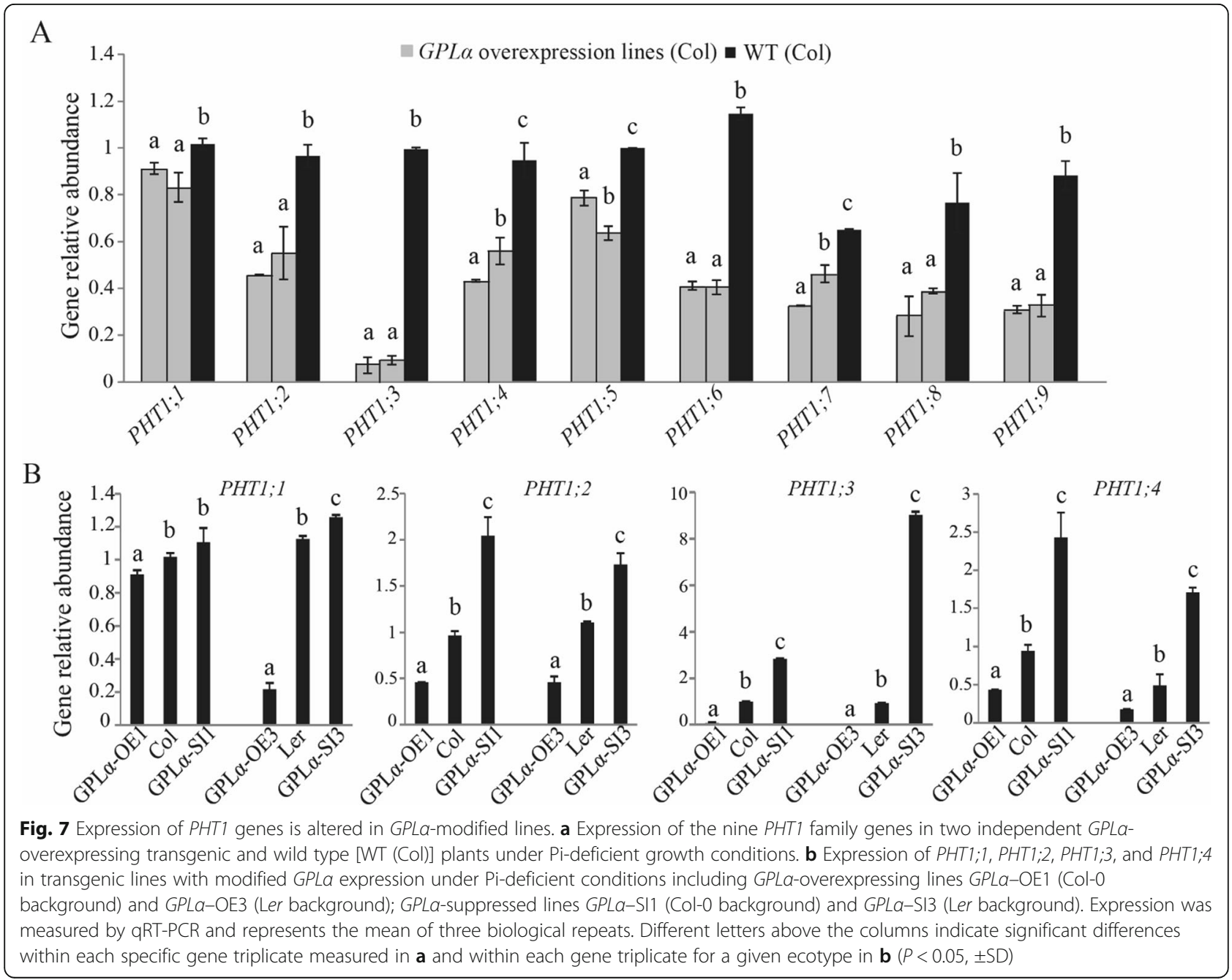


resulting in an increase in $\mathrm{Pi}$ and $\mathrm{P}$ levels [38]. To confirm this effect under our experimental conditions, both $\mathrm{Pi}$ and $\mathrm{P}$ contents were measured in leaves of Arabidopsis lines in which miR827 was either overexpressed or suppressed and which were grown hydroponically in Pi-sufficient media. Total $\mathrm{P}$ content increased to a large extent and more than doubled in two independent transgenic lines in which miR827 was overexpressed compared to that measured in the wild type (Fig. 8a). On the other hand, suppression of miR827 did not result in a decrease in $\mathrm{P}$ content in the miR827-suppressed lines compared to that measured for the wild type plants (Fig. 8a). Pi content increased about 2-fold in the miR827-overexpressing lines compared to the wild type, and miR827 suppression also had an impact on Pi content, resulting in a ca. $30 \%$ decrease (Fig. 8a). The effect of GPL $\alpha$ expression modification on P and $\mathrm{Pi}$ contents was studied in the two Arabidopsis accessions, Col-0 and Ler. In Col-0 GPL $\alpha$-overexpressing lines, total $\mathrm{P}$ levels were not significantly different from the wild type although they tended to be lower in the former (Fig. 8b). In the GPL $\alpha$-suppressed lines, including the two independent RNAi lines GPL $\alpha$-SI 1 and GPL $\alpha$-SI2 and the T-DNA mutant $G P L \alpha-K O 1$, the measured levels of total $\mathrm{P}$ were about $30-40 \%$ higher than those measured in the wild type (Fig. 8b). Similar measurements of Pi levels revealed about $40-50 \%$ reduction in the two GPL $\alpha$ overexpressing lines examined compared to the wild type, whereas in the three independent GPL $\alpha$-suppressed transgenic Arabidopsis Col-0 lines, Pi levels were about $25 \%$ higher than in the wild type (Fig. 8b). The same analyses were performed in the parallel transgenic Arabidopsis Ler accession in which GPL $\alpha$ expression was modified. Total P content was lower in the GPL $\alpha$-overexpressing plants compared to the wild type whereas in the three independent GPL $\alpha$-suppressed plants, P level was higher (Fig. 8c). Pi content in the GPL $\alpha$-suppressed Arabidopsis Ler plants was lower than that in the wild type, but higher than that of the wild type in the transgenic plants with suppressed expression (Fig. 8c).

\section{Discussion}

A marked number of senescence-regulated miRNAs have been reported to be nutrient-responsive [37]. In leaf senescence, nutrient recycling and remobilization to developing sink tissues are of major importance $[14,17]$. MiR408 and miR827, which exhibit the strongest induction in expression during leaf senescence, were subjected to further functional analyses. The study of miR408 revealed its significant involvement in Arabidopsis abiotic stress responses [67]. In the current study, we performed a detailed investigation of miR827.

\section{Effect of miR827-altered expression on Arabidopsis gene expression and leaf senescence}

NLA has been validated as a target of miR827 in Arabidopsis [42, 53]. NLA, a RING-type ubiquitin E3 ligase, can mediate ubiquitination and degradation of the plasma-membrane-localized PHT1 transporters, thereby negatively regulating their levels and affecting Pi homeostasis $[39,42]$. PHT5;1/VPT1, suggested to be a target of miR827 [42, 68], encodes an SPX domain protein that functions in Arabidopsis as a vacuolar Pi transporter shown to be essential for $\mathrm{Pi}$ homeostasis [54, 55]. In rice, both orthologs of PHT1;5/VPT1-OsSPX-MFS1 and OsSPX-MFS2were validated as targets of $m i R 827$, whereas no cleavage of the OsNLA transcript, which has a potential miR827 target, was detected [69]. In this study in Arabidopsis, we considered GPL $\alpha$ as an additional candidate target of miR827. GPL $\alpha$ is a member of the GeBP/GPL gene family, which encodes unconventional leucine-zipper transcription factors. Different members of this family have been reported to be involved in different developmental and stress responses [70-73].

Expression of NLA, PHT5;1/VPT1, and GPL $\alpha$ was found to be markedly induced when miR827 expression was also significantly induced (Fig. 3 ). We previously showed that while full-length $N L A$ transcript increases during late senescence, its putative miR827guided cleavage product increases even more strongly [37]. Thus, incoherent senescence regulation is observed for the NLA target gene with miR827, suggesting that miR827 moderates the target gene's expression during senescence rather than acting as an on/off switch.

Clear consequences to the target gene $N L A$, as well as to $P H T 5 ; 1 / V P T 1$ and GPL $\alpha$ transcript levels, were visualized following manipulation of pre-miR827 expression in transgenic plants. While the effect on $N L A$ transcript level was consistent with it being a target of miR827 as reported previously [39, 42], the consequences to GPL $\alpha$ expression support the notion that it may also be a target of miR827.

We examined the consequences of both inhibition and overexpression of pre-miR827. Overexpression of a premiRNA may not always be fully reflected in equivalently higher levels of the mature miRNA due to possible posttranscriptional processing events [31]. However, our measurements of pre-miR827 transcript levels during both senescence and $\mathrm{Pi}$ starvation were in agreement with the induction of the mature miR827 [37, 42]. Furthermore, the consequences of pre-miR827 
A

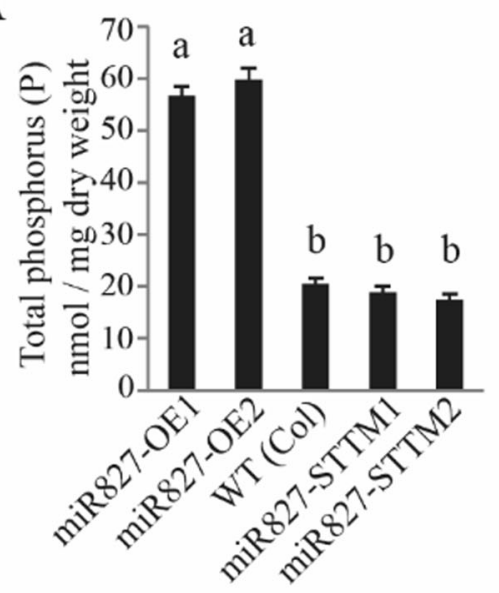

B
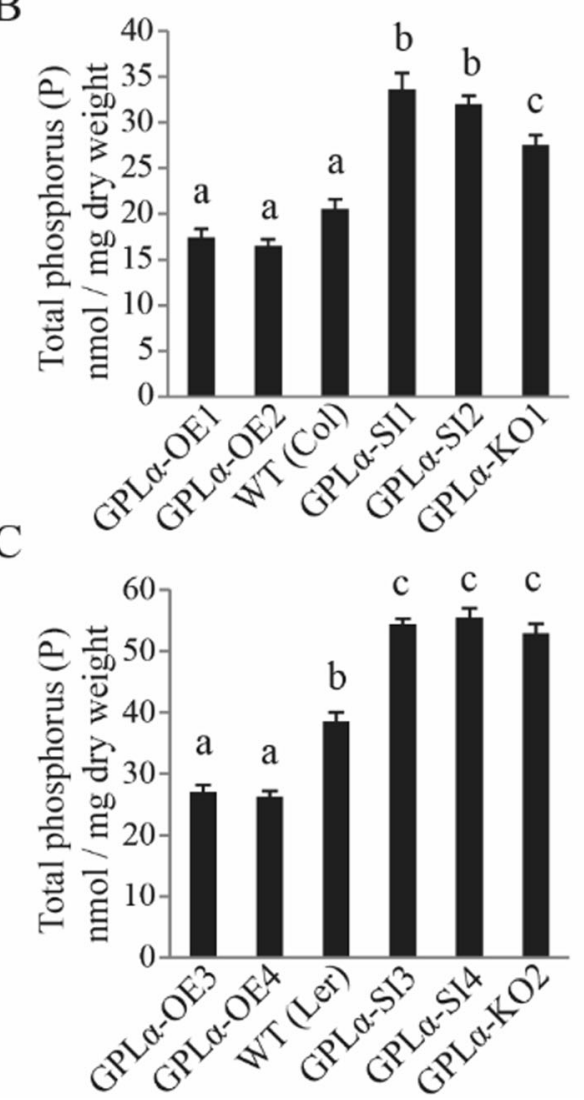
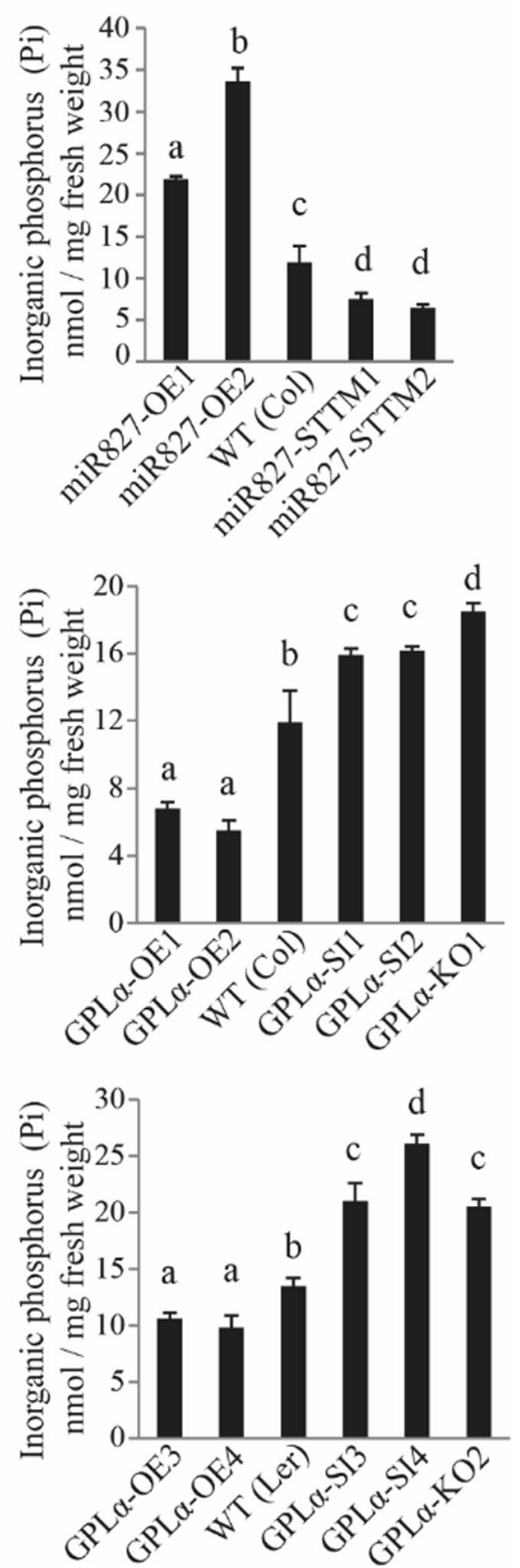

Fig. 8 Altered GPLa expression results in modified Pi levels in Arabidopsis leaves. Total phosphorus (P) and inorganic phosphorus (Pi) content was measured in leaves of miR827- and GPLa-modified plants grown hydroponically. The Pi and total P contents were measured in 28-day-old plants. a Left panel: total P measured in miR827-overexpressing lines (miR827-OE1 and 2), wild type (Col-0, WT), and miR827-suppressed lines (miR827STTM1 and 2). Right panel: Pi measured in these same miR827-modified lines. b Left panel: total P measured in GPLa-overexpressing lines (GPLaOE1 and 2), WT (Col-0), GPLa-suppressed lines (GPLa-SI1 and 2), and GPLa-mutant line (GPLa-KO1). Right panel: Pi measured in these same GPLamodified lines. c Left panel: total P measured in GPLa-overexpressing lines (GPLa-OE3 and 4), WT (Ler), GPLa-suppressed lines (GPLa-SI3 and 4), and GPLa-mutant line (GPLa-KO2). Right panel: Pi measured in these same GPLa-modified lines. Three independent transgenic lines were examined. Different letters above the columns indicate significant differences $(P<0.05, \pm$ SD $)$

overexpression for $N L A$ level and $\mathrm{P} / \mathrm{Pi}$ content found in our study (Fig. 3a and Fig. 8) were similar to those reported previously $[38,39,42]$.
The effects of pre-miR827 overexpression and inhibition on GPL $\alpha$ transcript level support the possibility that the latter is also a target of miR827. Further support 
stems from the inverse trends in the kinetics of GPL $\alpha$ and pre-miR827 expression under Pi-deficient growth conditions (Additional file 8: Figure S8). A potentially relatively close relationship was also discerned from the differential effect of altered miR827 expression on GPL $\alpha$ expression compared to its effect on other SAGs, including BFN1, involved in nuclear DNA degradation during late senescence $[58,74,75]$, and $S A G 12$, a cysteine protease with a role in $\mathrm{N}$ remobilization $[27,76]$. The level of GPL $\alpha$ expression was clearly more affected than those of BFN1 and SAG12 by altered miR827 expression.

Involvement of miR827 in the regulation of GPL $\alpha$ is also supported by the observed reduction in GPL $\alpha$ transcript level following transient co-expression with miR827, which was nullified by mutations in the predicted miR827-recognition site (Fig. 2). The pairing score of the predicted recognition site in GPL $\alpha$ was not the highest; however, it does fulfill the criteria suggested to be required for miRNA targets in Arabidopsis [77]. We were able to identify cleavage sites downstream of the predicted recognition site but not in the expected site within GPL $\alpha$. Identification of cleavage sites $3^{\prime}$ to the miRNA recognition site have been suggested to represent rapid and progressive transcript degradation [78, 79].

The effect of altered miR827 expression on senescence is likely the result of a combined function in regulating the expression of NLA and GPL $\alpha$. Mutant plants that were deficient in NLA displayed earlier onset of senescence compared to wild type plants. However, this enhanced-senescence phenotype was only observed under $\mathrm{N}$-limiting conditions and not under optimal growth conditions [80]. The involvement of NLA in senescence induced by $\mathrm{N}$ deficiency has been recently revealed [81]. ORE1, known to be key transcription factor regulating age-dependent leaf senescence in Arabidopsis $[82,83]$, was identified as a downstream target of NLA [81]. ORE1 protein stability was found to be controlled by the polyubiquitination-proteasome system involving NLA as an E3 ubiquitin ligase and PHO2/UBC24 as the partner E2 conjugase. It is likely that at least some of the consequences of miR827 overexpression for senescence acceleration, observed in our study, are mediated via NLA post-translational regulation of ORE1. Elevated miR827 expression resulted in reduced NLA, which in turn led to elevated ORE1 and acceleration of senescence (Fig. 9). Since in our study, the observed consequence of altered miR827 expression to senescence was observed under optimal growth conditions, it is suggested that regulation of ORE1 homeostasis by NLA/ miR827 operates not only under $\mathrm{N}$ deficiency-induced senescence, but is part of the delicate regulatory mechanism governing fine-tuning control of senescence. In addition, part of miR827s effect on leaf senescence could be attributed to its effect on GPL $\alpha$ expression. Induced expression of miR827 results in inhibited expression of GPL $\alpha$. Since GPL $\alpha$ has a negative regulatory effect, senescence is accelerated (Fig. 9).

Altered progress of senescence could result from developmental variability between plants, as well as from different environmental conditions. In this study, comparisons of the different mutant Arabidopsis lines were performed in parallel; thus, the different lines were subjected to the same growth conditions suggesting that the observed modifications in senescence progress are likely resulting from the genetic alterations in miR827 or GPL $\alpha$. We have not observed any consequences to overall plant development in the different lines investigated, which might have led to alterations in senescence initiation.

\section{GPLa is involved in regulation of senescence}

Functional analysis of GPL $\alpha$ indicated its possible involvement in senescence. Under optimal growth conditions, no apparent phenotypes were observed for plants with mutated GPL $\alpha$ expression. This fits well with $G P L \alpha$-specific expression in late senescence (Fig. 3). Other GeBP/GPL genes, including GeBP and GPL1, 2, and 3 , have been shown to be involved in a subset of cytokinin responses [70]. Overexpressing a version of GPL2 with constitutive transcriptional activation activity exhibited retarded growth, early senescence, and necrotic lesion phenotypes [73]. Phylogeny analysis of the Arabidopsis GeBP/GPL family members indicated that $G P L \alpha$ is distant from GeBP and GPL1, 2, and 3 [71]. Still, GPL $\alpha$ was found to have a repressive effect on senescence resembling the repressive-type function of the other described GeBP/GPL factors.

\section{Both miR827 and GPLa are involved in regulation of $\mathrm{Pi}$ homeostasis in Arabidopsis}

Previous research has provided ample evidence for miR827's involvement in $\mathrm{Pi}$ homeostasis [38, 39]. The observed induction of GPL $\alpha$ following exposure to Pi deficiency supports its involvement in $\mathrm{Pi}$ homeostasis as well; however, its late induction, 3-4 days after exposure to Pi deficiency, suggests its involvement in a rather late response to the stress compared to miR827. In this late P-response stage, when Pi deficiency becomes an extreme stress, a specific set of genes are induced [84] and regulation of Pi homeostasis, with GPL $\alpha$ involvement, might change.

Manipulation of miR827 expression resulted in altered contents of Pi and P (Fig. 8) as reported previously [38]. Interestingly, in both studies, the effect of miR827 overexpression on $\mathrm{P} / \mathrm{Pi}$ content was much more significant than that of miR827 inhibition. This suggests that reducing the level of NLA, which mediates degradation of 


\section{Senescence}
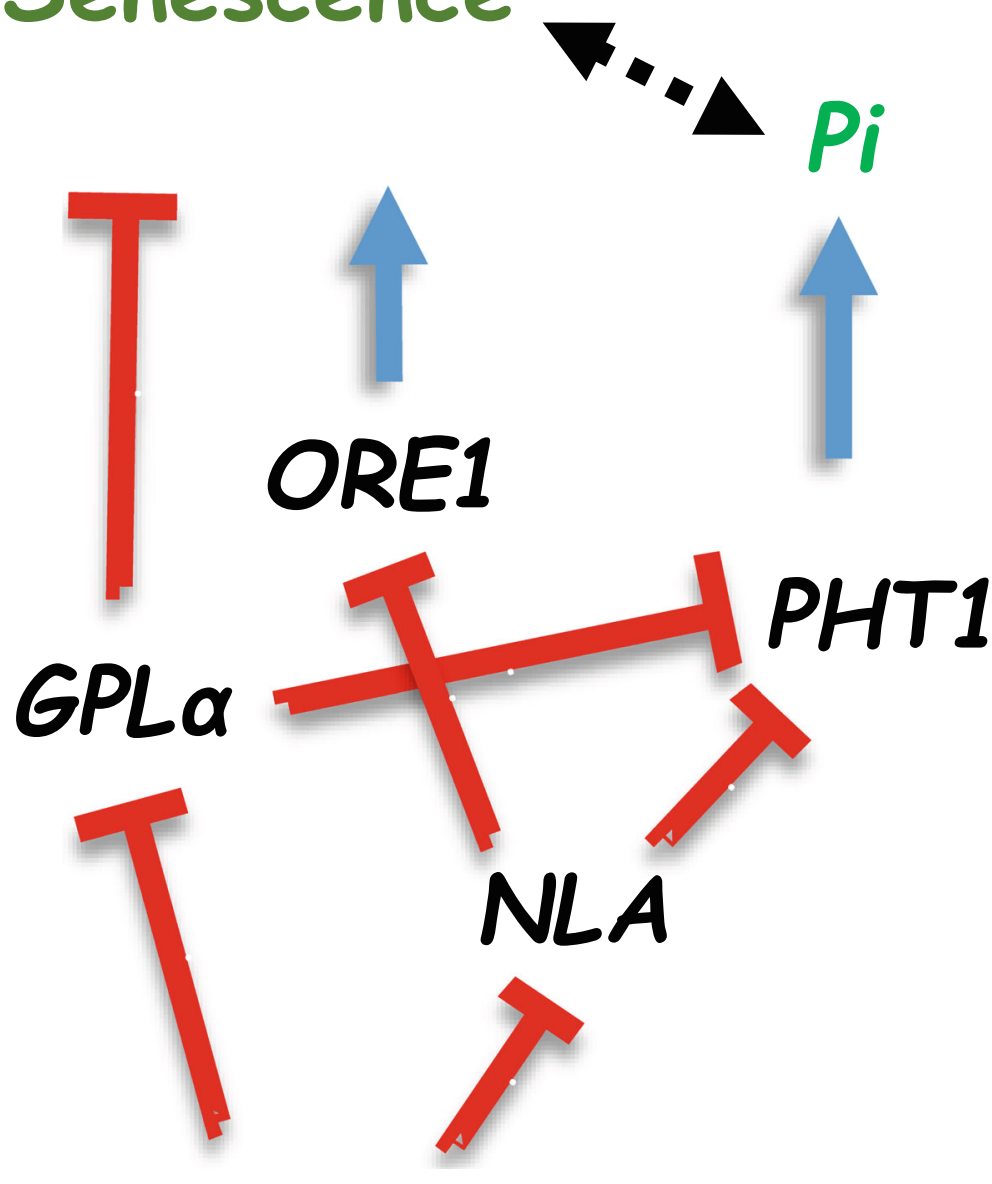

miR827

Fig. 9 Proposed model for the action of miR827 and GPLa. MiR827 controls the expression of PHT1 and Pi levels, by modulating the target NLA that negatively controls PHT1 protein level and in parallel, controlling GPLa expression, which also negatively controls PHT1 gene expression. Altered PHT1 levels affect tissue Pi levels. MiR827 controls the progression of senescence by modulating the target NLA that negatively controls protein levels of ORE1, which acts as a positive regulator of senescence, and in parallel modulating the expression of GPLa, which negatively controls senescence. MiR827 induction leads to increased PHT1 levels in parallel to its inducing effect on senescence via its effects on NLA and GPLa. Negative effect is indicated by red inhibitory signs and positive effect by blue arrows. Double-headed dashed black arrow indicates balanced changes

the PHT1 transporters, has a more prominent role in $\mathrm{Pi}$ homeostasis. Accordingly, a mutation in NLA results in a more significant effect of increased $\mathrm{P} / \mathrm{Pi}$ content compared to the effect of overexpressing NLA, which results in a mild reduction in $\mathrm{P} / \mathrm{Pi}$ content in Arabidopsis [38].

Altered expression of GPL $\alpha$ also had a clear but opposite effect on $\mathrm{P} / \mathrm{Pi}$ content. Overexpression of GPL $\alpha$ resulted in decreased $\mathrm{P} / \mathrm{Pi}$ levels, whereas suppression did not have a clear effect. Thus, GPL $\alpha$ seems to act as a negative regulator of $\mathrm{Pi}$ accumulation, similar to its negative effect on senescence.

Changes in response to Pi deficiency are well known to include a reduction in primary-root growth and an increase in density and length of the lateral roots and root hairs $[63,85,86]$. The consequences of altered expression of either GPL $\alpha$ or miR827 to root development further support the involvement of both genes in $\mathrm{Pi}$ homeostasis in Arabidopsis. The change in internal Pi level as a result of altered miR827 expression fits with the observed root-length phenotype. The opposite effect was observed on primary-root length in GPL $\alpha$-transgenic lines (Figs. 6 and 8). These results further support GPL $\alpha$ 's involvement in Pi homeostasis.

NLA directs degradation of the PHT1 transporters [87, 88], and miR827's function in $\mathrm{Pi}$ homeostasis is known to be mediated by regulation of $N L A$ transcript 
levels [38, 39]. GPL $\alpha$ was found in this study to be involved in the modulation of PHT1 expression (Fig. 7) with no effect on the expression of a few other examined Pi-starvation-related genes. This inhibitory effect of GPL $\alpha$ on PHT1 expression would explain the reduced levels of $\mathrm{P} / \mathrm{Pi}$ measured in the GPL $\alpha$-overexpressing lines.

Plant Pi transporters are known to be under complex regulation, by different transcription factors, such as MYB-, WRKY-, or BHLH-type involved in transcriptional regulation of PHT $[64,89]$. The central and important regulatory role of $G P L \alpha$ is suggested by its effect on the expression of all PHT1 genes. GPL $\alpha$ has a negative regulatory effect on $P H T 1$ gene expression as well as on $\mathrm{P} / \mathrm{Pi}$ content in the plant. Similar negative regulation on Pi-stress-induced responses in plants has been suggested for MYB62 [90].

Thus, a negative regulatory function of GPL $\alpha$ is suggested for both senescence and Pi-related responses. Other members of the GeBP/GPL gene family have been previously suggested to function as negative regulators: $G e B P$ was suggested to act as a repressor of leaf cell fate [71], and GPL1, GPL2, and GPL3 were shown to play a repressive role in the determination of final organ size and cell expansion [73]. These GPL genes have also been suggested to play a role in the cytokinin pathway mediated by their repressive function on Arabidopsis response regulators [70]. A recent investigation of GPL4 suggested its involvement in the roots' response to toxic metals [72]. Thus, although these different members of the $G e B P / G P L$ gene family seem to function in very different aspects of plant development and stress response, their mode of action seems to include a negative regulatory aspect, as observed in our study for the effect of GPL $\alpha$ on senescence and Pi homeostasis.

\section{Conclusions}

Our current study suggests a regulatory function for both miR827 and GPL $\alpha$ in Pi homeostasis as well as in senescence, as suggested in the model presented in Fig. 9. Induced expression of miR827 results in reduced expression of NLA which is known to negatively regulate $O R E 1$, thus resulting in accelerated senescence. At the same time, induced miR827 results in decreased GPL $\alpha$ expression, also associated with accelerated senescence. Thus, the function of miR827 for senescence induction could be mediated in parallel by its effects on either NLA or GPL $\alpha$. Interestingly, a similar parallel function of miR827 is proposed to affect Pi homeostasis via its negative regulatory effect on either $N L A$ or GPL $\alpha$, both having a negative regulatory effect on $P H T 1$ genes. Thus, miR827 activation enables increased Pi transport due to its effect on increasing PHT1 levels via its effects on both NLA and GPL $\alpha$. This suggested regulatory circuit involving miR827, GPL $\alpha, N L A$, and ORE1 could be part of the complex regulatory mechanism of senescence. Many studies support the view that the onset and progression of senescence are highly controlled, to enable efficient recycling of nutrients present in the leaves. $\mathrm{Pi}$ is an important nutrient that requires appropriate regulatory and functional components for its recycling, including the functioning of phosphate transporters such as PHT1s to enable export of the nutrient from the senescing tissue. The functions of miR827, NLA, GPL $\alpha, O R E 1$, and PHT1 and the interactions between them could be part of the mechanism controlling the balance between senescence progression, especially in its advanced stage, and recycling and transport of $\mathrm{Pi}$ for efficient nutrient recycling, which is the essence of senescence.

\section{Methods}

\section{Plant growth conditions}

Arabidopsis thaliana ecotypes Columbia (Col-0) and Landsberg erecta (Ler) were the wild type plants used. Two GPL $\alpha$ T-DNA insertional lines (ET2099.DS3.07.28.00.B.544, GK-252G05-014573) were ordered from the ABRC stock center. Seeds were germinated on soil or half-strength MS medium following 2 days of vernalization at $4{ }^{\circ} \mathrm{C}$. Plants were grown at $22^{\circ} \mathrm{C}$ under a $16 \mathrm{~h} / 8 \mathrm{~h}$ light/dark cycle. The complete medium contained the following: $2 \mathrm{mM} \mathrm{Ca}\left(\mathrm{NO}_{3}\right)_{2}, 2.5 \mathrm{mM}$ $\mathrm{KH}_{2} \mathrm{PO}_{4}, 1 \mathrm{mM} \mathrm{MgSO}_{4}, 5 \mathrm{mM} \mathrm{KNO}_{3}, 7 \mu \mathrm{M} \mathrm{H} \mathrm{H}_{3} \mathrm{BO}_{3}$, $50 \mu \mathrm{M} \mathrm{FeSO}, 50 \mu \mathrm{M}$ Na-EDTA, $14 \mu \mathrm{M} \mathrm{MnCl}_{2}, 0.7 \mu \mathrm{M}$ $\mathrm{ZnSO}_{4}, 0.2 \mathrm{mM} \mathrm{Na}_{2} \mathrm{MoO}_{4}$, and $50 \mathrm{nM} \mathrm{CuSO}$. To control Pi levels, $\mathrm{KH}_{2} \mathrm{PO}_{4}$ was replaced with $\mathrm{K}_{2} \mathrm{SO}_{4}[38,39]$. To examine the effects of Pi deficiency, 10-day-old seedlings were transferred to normal or Pi-deficient plates.

For experiments in which senescence was evaluated, either naturally or artificially induced senescing Arabidopsis plants were used. Single plants were grown in potting soil mixture Green 7611 (Green Ltd., http:// www.evenari.co.il) in $7 \mathrm{~cm} \times 7 \mathrm{~cm} \times 8 \mathrm{~cm}$ containers at $22{ }^{\circ} \mathrm{C}$ under a light/dark cycle of $16 / 8 \mathrm{~h}$ (long days). No fertilizer was added. For natural senescence, when initial yellowing tissue, indicative of senescence, was observed in one of the lines examined in a given experiment, leaves 5 and 6 were harvested from multiple plants, pooled, and frozen for further analyses. This usually occurred at about 35-45 days under our growth conditions which are similar to previously described kinetics of natural leaf senescence in Arabidopsis [22]. For artificially induced senescence experiments, Arabidopsis plants were grown as described above, until central stems were initiated. Intact plants were removed from the soil, their roots were excised, and they were placed in the dark, in containers fitted with inlet and outlet ports. The containers were sealed and connected to a flow-through system of air with a flow rate maintained at $50 \mathrm{~mL} / \mathrm{min}$, 
bubbled through sterile water to maintain humidity in the container. Once leaves 5 and 6 , in one of the lines, initiated senescence, they were harvested from multiple plants, pooled, and frozen for further experiments.

\section{Vector construction and plant transformation}

Full-length pre-miRNA of miR827 (miRBase accession no. MI0005383, TAIR accession no. AT3G59884) and GPL $\alpha$ (TAIR accession no. AT4G00610) were cloned from Arabidopsis. PCR amplification primers were designed by the software Primer Premier 6, and amplified PCR fragments were cloned into the XhoI-XbaI (miR827) or NcoI-XbaI (GPLa) restriction sites of the plant expression vector $p F G C 5941$ (GenBank accession no. AY310901), resulting in vectors $p F G C 5941-p 35 S$ miR827 and pFGC5941-p35S-GPL, respectively. STTM strategy was used to silence miR827 [91]. The STTM sequence was synthesized (GENEWIZ) and cloned into the $p F G C 5941$ vector restriction sites $N c o I-X b a \mathrm{I}$, resulting in vector $p F G C 5941-p 35 S-827 S T T M$. For the GPL $\alpha$ silencing vector, a segment of the GPL $\alpha$ cDNA sequence was PCR amplified and cloned as two inverted repeats into the restriction sites $\mathrm{XhoI}-\mathrm{Nco} \mathrm{I}$ and $\mathrm{XbaI}-\mathrm{BamHI}$ of vector $p F G C 5941$, resulting in RNAi vector $p F G C 5941$ p35S-GPL-SI.

Genomic Arabidopsis DNA, including the 1325-bp 5' sequence of the miR827 promoter or the 2030-bp sequence of GPL $\alpha$ up to ATG, were cloned into restriction sites SalI-NotI, of the pORE R4 vector (ordered from TAIR) [92], resulting in vector $p F G C$-5941-p827-GFP and $p F G C-5941-p G P L-G F P$, respectively. For subcellular localization analysis, GPL $\alpha$ cDNA, including the coding sequences, was cloned upstream or downstream of the GFP cDNA coding sequences to generate either $\mathrm{N}$ - or C-terminal translational fusions of GPL $\alpha$ and GFP coding sequences (GPL $\alpha-G F P$, GFP-GPL $\alpha$ ) using the Gateway cloning system (Invitrogen, pDONR/SD/D TOPO and pK7FWG2.0, pK7FWF2.0 vectors). Chimeric genes were cloned under the $35 S$ promoter. All of the primers are listed in Additional file 13: Table S1.

For construction of vectors for transient expression, ath-pre-miR827 and the full-length GPL $\alpha$ cDNA coding sequences were used, as well as those of grape $V v i$ miR171d and $V v$-SCL15. The predicted miR827 target site in GPL $\alpha$ was mutated at the coding sequence nucleotide positions 792 and 795, replacing T with C (Additional file 3: Figure S3). The pre-miR827 or pre-VvimiR171d were cloned into the vector pGreen II-62-SK (Youbio, China), while GPL $\alpha$ or SCL15 coding sequences were cloned into the vector pGreen II-0800-miRNA (Youbio). All cDNAs were cloned under the $35 S$ promoter.

Transformation of Arabidopsis plants was performed by Agrobacterium-mediated floral dip method [93].
Homozygous lines were established and T3 or T4 lines were used for the experiments. Experiments designed to examine the effects of miR827 on GPL $\alpha$ were performed by transient co-expression of the relevant expression vectors following Agrobacterium infiltration of Nicotiana benthamiana leaves. The expression vectors were cotransformed with the helper pSoup19 into Agrobacterium strain GV3101 used for tobacco-infiltration experiments. The bacteria were suspended in infiltration medium (10 mM $\mathrm{MgCl}_{2}, 10 \mathrm{mM}$ MES-KOH, pH 5.2, 0.1 $\mathrm{mM}$ acetosyringone) at $\mathrm{OD}_{600}=1$, and incubated for 4$5 \mathrm{~h}$ at room temperature. The bacterial suspension was injected into the leaves with a syringe. Zones of infiltrated tissues were harvested 4 days after injection for RNA isolation.

\section{Protein and chlorophyll content measurements}

Arabidopsis leaf was extracted with $150 \mu \mathrm{L}$ extraction buffer $(50 \mathrm{mM}$ Tris- $\mathrm{HCl} \mathrm{pH}$ 7.5, 0.1\% w/v SDS, $10 \mathrm{mM}$ EDTA, and $1 \mathrm{mM}$ PMSF) by crushing in a 2010 Geno Grinder (SPEX Sample Prep). For the chlorophyll assay, $170 \mu \mathrm{L} \mathrm{H}_{2} \mathrm{O}$ was added to $30 \mu \mathrm{L}$ of the extract, followed by vortexing and extraction with $800 \mu \mathrm{L}$ acetone. Following $10 \mathrm{~min}$ centrifugation at maximal speed, the supernatant was used to measure absorbance for quantification of chlorophyll $\mathrm{a}+\mathrm{b}$ [94]. For protein concentration measurements, the remaining extract was centrifuged at $18,000 \mathrm{~g}$ at room temperature for $10 \mathrm{~min}$ and the clear supernatant was used to measure protein content with a protein assay kit (BioRad).

\section{Quantitative RT-PCR}

Total RNA was isolated using the Spectrum Plant Total RNA kit (Sigma-Aldrich) and RNA was reversetranscribed with the Verso cDNA Synthesis kit (Thermo Scientific) using gene-specific primers or oligo(dT). The qRT-PCR was performed with a StepOne ${ }^{\mathrm{rm}}$ Real-Time PCR System (Applied Biosystems) using Fast SYBR Green Master Mix (Applied Biosystems) and genespecific primers (Additional file 13: Table S2). The qRTPCR primers were designed with Primer Express 3.0 software (Applied Biosystems). Expression data were analyzed by CT (cycle threshold) value $[95,96]$. All experiments were carried out with non-template controls and in three biological repeats.

\section{Transient expression in Arabidopsis and confocal microscopy fluorescence observations}

Transient expression in Arabidopsis was performed for localization experiments using the vectors harboring the GPL $\alpha$-GFP and GFP-GPL $\alpha$ translational fusions, as well as the GFP included in the pFGC5941 vector as a control. Vectors were transformed into Agrobacterium tumefaciens strain EHA105 by the heat-shock 
transformation method. Agrobacterium leaf infiltration was performed as described previously [97]. Confocal microscopy observation and image acquisition were carried out with an Olympus IX 81 inverted confocal laser scanning microscope (FLUOVIEW 500) equipped with a 488-nm argon-ion laser and 60X 1.0 NA Plan Apo water-immersion objective. GFP was excited by $488 \mathrm{~nm}$ light and the emission was collected through a BA 515525 filter; a BA 660 IF emission filter was used to collect chlorophyll autofluorescence. Confocal optical sections were obtained at $0.5-\mu \mathrm{m}$ increments.

\section{Fluorescence imaging}

GFP fluorescence imaging of leaves of transgenic plants was performed using the IVIS Lumina II imaging system (PerkinElmer). To measure GFP fluorescence, appropriate filters were used to obtain $430 \mathrm{~nm}$ excitation and $530 \mathrm{~nm}$ emission. The conditions used for imaging included $12.5 \mathrm{~cm}$ field of view for sample, 1.2 lens aperture $\mathrm{f} / \mathrm{stop}$, and medium pixel binning (CCD resolution) (to balance pixel size and sensitivity). Acquisition time was 3-5 s. Data capture and analysis were performed using IVIS Lumina II Living Image ${ }^{\bullet}$ Software.

\section{Quantification of total P and soluble Pi}

Total P and soluble Pi contents were analyzed as described previously $[98,99]$ with minor modifications. Total P quantification was performed using leaf tissue dried at $80^{\circ} \mathrm{C}$ for $24 \mathrm{~h}$. A 100 -mg sample of dry leaf material was dissolved in $2 \mathrm{~mL}$ sulfuric acid and digested in a heat block at $250^{\circ} \mathrm{C}$ for $1 \mathrm{~h}$. After cooling to room temperature, $300 \mu \mathrm{L} \mathrm{H}_{2} \mathrm{O}_{2}$ was added to the solution; the solution was heated to $250{ }^{\circ} \mathrm{C}$ for 10 min and then cooled to room temperature. These steps were repeated until the solution was clear. The solution was diluted 50 -fold in water and used to measure P concentration. For soluble Pi measurement, fresh tissue was crushed in liquid $\mathrm{N}$ and homogenized with $1 \%$ glacial acetic acid. Then, $50 \mu \mathrm{L}$ sample was mixed with $250 \mu \mathrm{L}$ water and $700 \mu \mathrm{L}$ assay buffer $\left(600 \mu \mathrm{L}\right.$ solution of $0.42 \% \mathrm{w} / \mathrm{v} \mathrm{NH}_{4} \mathrm{MoO}_{4}$ and $0.86 \mathrm{~N}$ $\mathrm{H}_{2} \mathrm{SO}_{4}, 100 \mu \mathrm{L} 10 \%$ ascorbic acid) and incubated at $42^{\circ} \mathrm{C}$ for $20 \mathrm{~min}$. Pi content was assayed by measuring absorbance at $820 \mathrm{~nm}$.

\section{Accession numbers}

Sequence data from this article can be found in The Arabidopsis Information Resource (TAIR) or the National Center for Biotechnology Information (NCBI) under the following Arabidopsis Genome Initiative (AGI) locus identifiers: miR827 (At3g59884), GPL $\alpha$ (At4g00610), NLA (At1g02860), SPX (At1g63010), SAG12 (At5g45890), BFN1 (At1g11190), PHO1 (At3g23430), PHO2 (At2g33770), PHT1;1 (At5g43350),
PHT1;2 (At5g43370), PHT1;3 (At5g43360), PHT1;4 (At2g38940), PHT1;5 (At2g32830), PHT1;6 (At5g43340), PHT1;7 (At3g54700), PHT1;8 (At1g20860), PHT1;9 (At1g76430), PHT2 (At3g26570), PHF1 (At3g52190), AtIPK1 (At5g42810), AtPAP12 (At2g27190), AtPAP26 (At5g34850), ZAT6 (At5g04340), ACTIN (AtT3g18780), and $U B C$ (At5g25760).

\section{Abbreviations}

GPLa: GLABRA1 enhancer-binding protein (GeBP)-like; SAG: Senescenceassociated gene; miRNA: MicroRNA; NLA: Nitrogen limitation adaptation; PHT1: Phosphate transporter 1; Col-0: Columbia; Ler: Landsberg erecta

\section{Supplementary Information}

Supplementary information accompanies this paper at https://doi.org/10. 1186/s12915-021-01015-2.

Additional file 1: Fig. S1. Expression of pre-miR827 is induced during natural and dark-induced leaf senescence and is regulated by upstream sequences. $A$ and $C$ Expression of pre-miR827 during natural senescence (A) and artificial dark-induced senescence (C) was measured by qRT-PCR in young $(Y)$, mature $(M)$, early senescence (ES) and late senescence (LS) leaves, and in late-stage dark-induced senescence (DIS). Different letters and asterisk indicate significant difference $(P<0.05$, Student's $t$ test, \pm SD). $B$ and D GFP fluorescence measured in leaves of transgenic plants containing miR827-promoter-driven GFP (miR827Pro:GFP) during natural senescence (B) or dark-induced senescence (D). Six independent transgenic lines were examined and representative lines are shown. WT, wild type.

Additional file 2: Fig. S2. Vector constructs and effects of genetic manipulations of pre-miR827 expression level on transgenic plants. A Upper map: transformation vector constructed for overexpression of miR827. The precursor of miR827 is regulated by the constitutive 355 promoter. Lower map: transformation vector constructed for silencing of miR827 using a target mimic method (STTM) to silence miR827 activated constitutively by control of the 355 promoter. Construction details are described in Methods. B Expression levels of pre-miR827 in two independent transgenic lines overexpressing miR827 (35S:827-OE1, 2), the wild type [WT $(\mathrm{Col})]$, and two independent miR827-silenced lines (35S:827-STTM1, 2). Expression was measured by qRT-PCR and represents the mean of three biological repeats; values were normalized to WT levels. Asterisk indicates significant difference from WT $(P<0.05$, Student's $t$ test, \pm SD).

Additional file 3: Fig. S3. Predicted miR827-recognition site in the GPLa sequence. Upper row, sequence of GPLa transcript from nucleotides 778825. Sequence identified as putative target for miR827 is labeled in red and the extent of base-pairing to the miR827 sequence shown in the lower row is presented. Two $U$ positions changed to $C$ in the mutant version of GPLa are shown in blue above the recognition site. Cleavage-site position experimentally identified by 5' RACE analysis for 5 out of 13 incidences examined is indicated by a vertical arrow. Another 8 sites were spread among 5 additional locations in the 100-bp region downstream of the putative recognition site.

Additional file 4: Fig. S4. Senescence-associated expression of GPLa is altered in transgenic plants with modified miR827 expression. Expression levels of GPLa, SAG12 and BFN1 were measured by qRT-PCR in leaves of miR827-overexpressing (miR827-OE1), wild-type (WT) (Col-0) and miR827silenced (miR827-STTM1) lines at different senescence stages. Relative abundance of the transcripts of the three genes was plotted against chlorophyll content, representing senescence stage. Three independent transgenic lines were examined. Error bars correspond to \pm SD. FW, fresh weight.

Additional file 5: Fig. S5. Expression of GPLa is induced during natural and dark-induced leaf senescence and is regulated by upstream sequences. A and C Expression of GPLa during natural senescence (A) and artificial dark-induced senescence (C) was measured using GRT-PCR in young $(Y)$, mature $(M)$, early senescence (ES), and late senescence (LS) 
leaves, and in late dark-induced senescence (DIS). Different letters and asterisk indicate significant difference $(P<0.05$, Student's $t$ test, \pm SD). B and D GFP fluorescence measured in leaves of transgenic plants containing GPLa-promoter-driven GFP (GPLaPro:GFP) during natural senescence (B) or dark-induced senescence (D). Three independent transgenic lines were examined and representative lines are shown. WT, wild type.

Additional file 6: Fig. S6. Vector constructs and effects of genetic manipulations of GPLa expression level on transgenic plants. A Upper map: transformation vector constructed for overexpression of GPLa. Fulllength GPLa coding sequence was cloned and regulated by the constitutive 355 promoter. Middle panel: map of the constructed transformation vector for silencing GPLa. The RNAi gene constructed to silence GPLa was regulated by the constitutive 355 promoter. Construction details are described in Methods. Lower map: genomic sites of T-DNA insertions for the two independent GPLa-mutant lines-ET2099.Ds3.07.28.00.6.544 (GPLa-KO1) and GK-252G05-014573 (GPLa-KO2). B-D Expression levels of GPLa in transgenic plants overexpressing GPLa (GPLa OE1, 2), or with silenced GPLa (GPLa SI1, 2), and in the wild type [WT (Col)] (B), and in transgenic plants GPLa OE3, 4, GPLa SI3, 4, and WT (Ler) (C). Expression levels of GPLa in the GPLa mutants in the two ecotypes (GPLa KO1 in Col-0, GPLa KO2 in Ler) (D). Expression was measured by qRT-PCR and represents the mean of three biological repeats. Asterisk indicates significant difference from $W T(P<0.05$, Student's $t$ test, \pm SD).

Additional file 7: Fig. S7. Altered expression of GPLa in transgenic plants results in modified progression of leaf senescence. A and D Inhibited senescence in transgenic plants (Ler background) overexpressing GPLa (GPLa OE3) during natural leaf senescence (A, left panel) and artificial dark-induced senescence ( $D$, left panel). Accelerated senescence in GPLa-silenced transgenic plants (GPLa SI3) during natural leaf senescence (A, middle panel) and artificial dark-induced senescence ( $\mathrm{D}$, middle panel). Accelerated senescence in GPLa-mutant plants (GPLa $\mathrm{KO} 2$ ) during natural leaf senescence (A, right panel) and artificial darkinduced senescence ( $D$, right panel). WT (Ler), wild-type Ler accession. B and C Effects of GPLa overexpression or suppression on total chlorophyll (B) or protein (C) contents during natural leaf senescence. E and F Effects of overexpression or suppression of GPLa expression on total chlorophyll (E) and protein (F) contents during dark-induced leaf senescence. Three independent transgenic lines were examined. Asterisks indicate significant differences within each compared pair $(P<0.05$, Student's $t$ test, \pm $\mathrm{SD})$

Additional file 8: Fig. S8. Induction of pre-miR827, GPLa and PHT1 expression following exposure to Pi-deficient growth conditions. A and B Wild-type (Col-0) seedlings (10 days old) were transferred to Pi-deficient medium and expression was measured simultaneously in leaves (A) and roots (B) at different times after transfer. The primers used to measure PHT1 gene expression enabled the measurement of the total transcript of a few different members of the PHT1 gene family. The primers (Table S2) fully matched PHT1;1 and PHT1;2 and likely recognized a few additional PHT1 members with some mismatches in the primer sequences. Error bars indicate $\pm S D$

Additional file 9: Fig. S9. GPLa-GFP is localized to the nuclei. Leaves were transiently transformed with a vector including a translational fusion between GPLa and GFP, 35s:GFP::GPLa. GFP protein localization was visualized using confocal microscopy. (a) GFP fluorescence; (b) DAPI nuclei staining dye fluorescence; (c) bright-field image; (d) overlay of $a$, b, and c

Additional file 10: Fig. S10. Measured root length for the mutant line GPLa-KO2 in which GPLa was overexpressed [GPLa-OE (GPLa-KO2)]. The mutant line GPLa-KO2 (Ler background) was transformed for overexpression of GPLa and the consequences for root length following exposure to growth under Pi deficiency were examined in three independent transgenic lines. Different letters above the columns indicate significant differences $(P<0.05, \pm \mathrm{SD})$. WT, wild type.

Additional file 11: Fig. S11. Expression of $P H T 1$ and $P H O 1$ is induced under Pi-deficient conditions. Expression of PHT1 (A) and PHO1 (B) was measured in both Col-0 (Col MS) and Ler (Ler MS) wild-type accessions following transfer of seedlings to Pi-deficient growth conditions for 7 days (Col MS-Pi and Ler MS-Pi, respectively). Expression was measured by qRT-PCR and represents the mean of three biological repeats. Asterisks indicate significant difference $(P<0.05$, Student's t-test, \pm SD). The primers used to measure PHT1 expression were as described for Additional file 8.

Additional file 12: Fig. S12. Expression analysis of Pi-deficiencyresponsive genes in GPLa-overexpressing lines. Expression was measured by qRT-PCR and represents the mean of three biological repeats. Different letters above the columns indicate significant differences $(P<0.05, \pm$ SD). WT, wild type.

Additional file 13: Table S1. Sequences of primers used in gene cloning and vector construction. Table S2. Primers used for GRT-PCR analysis of gene expression.

\section{Acknowledgements}

C.M. was a recipient of the ARO (Agricultural Research Organization)-Israel Postdoctoral Fellowship. We are very grateful to Sarit Vilenchik for her technical assistance in some aspects of this research.

\section{Authors' contributions}

Research was conceived by C.M. and A.L. Experimental work was carried out and data analyzed by C.M., Q.C., and S.W. Research was supervised by A.L. Manuscript preparation was carried out by C.M and A.L. All authors read and approved the final manuscript.

\section{Funding}

This research was supported by grant no. IS-4180-08 from BARD, the United States-Israel Binational Agricultural Research and Development Fund to A.L.

Availability of data and materials

All data generated or analyzed during this study are included in this published article and its supplementary information files.

\section{Declarations}

Ethics approval and consent to participate

Not applicable.

\section{Consent for publication}

Not applicable.

\section{Competing interests}

The authors declare that they have no competing interests.

\section{Author details}

'Department of Plant Science, School of Agriculture and Biology, Shanghai Jiao Tong University, Shanghai 200240, China. ${ }^{2}$ Department of Postharvest Science, Agricultural Research Organization, Volcani Center, HaMaccabim Road 68, 7505101 Rishon LeZion, Israel.

Received: 16 June 2020 Accepted: 29 March 2021

Published online: 03 May 2021

\section{References}

1. Guo Y, Gan S. Leaf senescence: signals, execution, and regulation. Curr Top Dev Biol. 2005;71:83-112. https://doi.org/10.1016/S0070-2153(05)71003-6.

2. Thomas H, Ougham HJ, Wagstaff $C$, Stead AD. Defining senescence and death. J Exp Bot. 2003;54(385):1127-32. https://doi.org/10.1093/jxb/erg133.

3. Woo HR, Kim HJ, Lim PO, Nam HG. Leaf senescence: systems and dynamics aspects. In: Merchant SS, editor. Annual Review of Plant Biology, Vol 70; 2019. p. 347-76.

4. Hörtensteiner S. Chlorophyll degradation during senescence. Annu Rev Plant Biol. 2006;57(1):55-77. https://doi.org/10.1146/annurev.arplant.57.032 905.105212

5. Thomas $\mathrm{H}$. Senescence, ageing and death of the whole plant. New Phytol. 2013;197(3):696-711. https://doi.org/10.1111/nph.12047.

6. Kim J, Kim JH, Lyu JI, Woo HR, Lim PO. New insights into the regulation of leaf senescence in Arabidopsis. J Exp Bot. 2018:69(4):787-99. https://doi. org/10.1093/jxb/erx287.

7. Thomas $\mathrm{H}$, Huang $\mathrm{L}$, Young $\mathrm{M}$, Ougham $\mathrm{H}$. Evolution of plant senescence. BMC Evol Biol. 2009;9(1):163. https://doi.org/10.1186/1471-2148-9-163. 
8. Lers A. Environmental regulation of leaf senescence. In: Gan S, editor. Senescence Processes in Plants. Oxford: blackwell Publishing; 2007. p. 10833.

9. Sade N, Rubio-Wilhelmi MD, Umnajkitikorn K, Blumwald E. Stress-induced senescence and plant tolerance to abiotic stress. J Exp Bot. 2018;69(4):84553. https://doi.org/10.1093/jxb/erx235.

10. Fageria NK, Baligar VC, Li YC. The role of nutrient efficient plants in improving crop yields in the twenty first century. J Plant Nutr. 2008;31(6): 1121-57. https://doi.org/10.1080/01904160802116068.

11. Diaz C, Lemaître T, Christ A, Azzopardi M, Kato Y, Sato F, Morot-Gaudry JF, le Dily F, Masclaux-Daubresse $C$. Nitrogen recycling and remobilization are differentially controlled by leaf senescence and development stage in Arabidopsis under low nitrogen nutrition. Plant Physiol. 2008;147(3):1437-49. https://doi.org/10.1104/pp.108.119040

12. Fischer AM. Nutrient remobilization during leaf senescence. Annual Plant Reviews Volume 26: Senescence Processes in Plants. Oxford: Blackwell Publishing Ltd; 2007. p. 87-107.

13. Gregersen PL, Holm PB, Krupinska K. Leaf senescence and nutrient remobilisation in barley and wheat. Plant Biol. 2008;10:37-49.

14. Himelblau E, Amasino RM. Nutrients mobilized from leaves of Arabidopsis thaliana during leaf senescence. J Plant Physiol. 2001;158(10):1317-23. https://doi.org/10.1078/0176-1617-00608.

15. Hörtensteiner S, Feller U. Nitrogen metabolism and remobilization during senescence. J Exp Bot. 2002;53(370):927-37. https://doi.org/10.1093/jexbot/ 53.370 .927$.

16. Liu J, Wu YH, Yan JJ, Liu YD, Shen FF. Protein degradation and nitrogen remobilization during leaf senescence. J Plant Biol. 2008;51(1):11-9. https:// doi.org/10.1007/BF03030735.

17. Guiboileau A, Sormani R, Meyer C, Masclaux-Daubresse C. Senescence and death of plant organs: nutrient recycling and developmental regulation. C R Biol. 2010;333(4):382-91. https://doi.org/10.1016/j.crvi.2010.01.016.

18. Avice J-C, Etienne P. Leaf senescence and nitrogen remobilization efficiency in oilseed rape (Brassica napus L.). J Exp Bot. 2014;65(14):3813-24. https:// doi.org/10.1093/jxb/eru177.

19. Maillard A, Diquelou S, Billard V, Laine P, Garnica M, Prudent M, et al. Leaf mineral nutrient remobilization during leaf senescence and modulation by nutrient deficiency. Front Plant Sci. 2015;6 https://doi.org/10.3389/fpls.2015. 00317.

20. Leopold AC. Senescence in Plant Development: The death of plants or plant parts may be of positive ecological or physiological value. Science. 1961;134:1727-32. https://doi.org/10.1126/science.134.3492.1727.

21. Balazadeh S, Riano-Pachon DM, Mueller-Roeber B. Transcription factors regulating leaf senescence in Arabidopsis thaliana. Plant Biol. 2008;10:63-75.

22. Breeze E, Harrison E, McHattie S, Hughes L, Hickman R, Hill C, Kiddle S, Kim YS, Penfold CA, Jenkins D, Zhang C, Morris K, Jenner C, Jackson S, Thomas B, Tabrett A, Legaie R, Moore JD, Wild DL, Ott S, Rand D, Beynon J, Denby K, Mead A, Buchanan-Wollaston V. High-resolution temporal profiling of transcripts during Arabidopsis leaf senescence reveals a distinct chronology of processes and regulation. Plant Cell. 2011;23(3):873-94. https://doi.org/1 0.1105/tpc.111.083345.

23. Wagstaff C, Yang TJW, Stead AD, Buchanan-Wollaston V, Roberts JA. A molecular and structural characterization of senescing Arabidopsis siliques and comparison of transcriptional profiles with senescing petals and leaves. Plant J. 2009;57(4):690-705. https://doi.org/10.1111/j.1365-313X.2008.03722.x.

24. Kim HJ, Nam HG, Lim PO. Regulatory network of NAC transcription factors in leaf senescence. Curr Opin Plant Biol. 2016;33:48-56. https://doi.org/10.1 016/j.pbi.2016.06.002

25. Schippers JHM. Transcriptional networks in leaf senescence. Curr Opin Plant Biol. 2015;27:77-83. https://doi.org/10.1016/j.pbi.2015.06.018.

26. Hanfrey C, Fife M, BuchananWollaston V. Leaf senescence in Brassica napus: expression of genes encoding pathogenesis-related proteins. Plant Mol Biol. 1996;30(3):597-609. https://doi.org/10.1007/BF00049334.

27. Noh YS, Amasino RM. Identification of a promoter region responsible for the senescence-specific expression of SAG12. Plant Mol Biol. 1999;41(2):18194. https://doi.org/10.1023/A:1006342412688

28. Oh SA, Lee SY, Chung IK, Lee CH, Nam HG. A senescence-associated gene of Arabidopsis thaliana is distinctively regulated during natural and artificially induced leaf senescence. Plant Mol Biol. 1996;30(4):739-54. https://doi.org/10.1007/BF00019008.

29. Chen W, Provart NJ, Glazebrook J, Katagiri F, Chang H-S, Eulgem T, Mauch F, Luan S, Zou G, Whitham SA, Budworth PR, Tao Y, Xie Z, Chen X, Lam S,
Kreps JA, Harper JF, Si-Ammour A, Mauch-Mani B, Heinlein M, Kobayashi K Hohn T, Dangl JL, Wang X, Zhu T. Expression profile matrix of arabidopsis transcription factor genes suggests their putative functions in response to environmental stresses. Plant Cell. 2002;14(3):559-74. https://doi.org/10.11 05/tpc.010410.

30. Swida-Barteczka A, Szweykowska-Kulinska Z. Micromanagement of developmental and stress-induced senescence: the emerging role of microRNAs. Genes. 2019;10(3):210. https://doi.org/10.3390/genes10030210.

31. Szweykowska-Kulinska Z, Jarmolowski A. Post-transcriptional regulation of microRNA accumulation and function: new insights from plants. Mol Plant. 2018;11(8):1006-7. https://doi.org/10.1016/j.molp.2018.06.010.

32. Kim JH, Woo HR, Kim J, Lim PO, Lee IC, Choi SH, Hwang D, Nam HG. Trifurcate feed-forward regulation of age-dependent cell death involving miR164 in Arabidopsis. Science. 2009;323(5917):1053-7. https://doi.org/1 $0.1126 /$ science.1166386.

33. Schommer C, Palatnik JF, Aggarwal P, Chetelat A, Cubas P, Farmer EE, et al. Control of jasmonate biosynthesis and senescence by miR319 targets. Plos Biol. 2008;6(9):1991-2001.

34. Huo $X Y$, Wang $C$, Teng $Y B$, Liu $X Y$. Identification of miRNAs associated with dark-induced senescence in Arabidopsis. BMC Plant Biol. 2015;15(1):266. https://doi.org/10.1186/s12870-015-0656-5.

35. Woo HR, Koo HJ, Kim J, Jeong H, Yang JO, Lee IH, Jun JH, Choi SH, Park SJ, Kang B, Kim YW, Phee BK, Kim JH, Seo C, Park C, Kim SC, Park S, Lee B, Lee S, Hwang D, Nam HG, Lim PO. Programming of plant leaf senescence with temporal and inter-organellar coordination of transcriptome in arabidopsis. Plant Physiol. 2016;171(1):452-67. https://doi.org/10.1104/pp.15.01929.

36. Xu XB, Bai HQ, Liu CP, Chen EY, Chen QG, Zhuang JY, et al. Genome-wide analysis of microRNAs and their target genes related to leaf senescence of rice. Plos One. 2014;9(12). https://doi.org/10.1371/journal.pone.0114313.

37. Thatcher SR, Burd S, Wright C, Lers A, Green PJ. Differential expression of miRNAs and their target genes in senescing leaves and siliques: insights from deep sequencing of small RNAs and cleaved target RNAs. Plant Cell Environ. 2015;38(1):188-200. https://doi.org/10.1111/pce.12393.

38. Kant S, Peng MS, Rothstein SJ. Genetic regulation by NLA and microRNA827 for maintaining nitrate-dependent phosphate homeostasis in Arabidopsis. Plos Genet. 2011;7(3). https://doi.org/10.1371/journal.pgen.1002021.

39. Lin WY, Huang TK, Chiou TJ. Nitrogen limitation adaptation, a target of microRNA827, mediates degradation of plasma membrane-localized phosphate transporters to maintain phosphate homeostasis in Arabidopsis. Plant Cell. 2013;25(10):4061-74. https://doi.org/10.1105/tpc.113.116012.

40. Liu TY, Lin WY, Huang TK, Chiou TJ. MicroRNA-mediated surveillance of phosphate transporters on the move. Trends Plant Sci. 2014;19(10):647-55. https://doi.org/10.1016/j.tplants.2014.06.004.

41. Nguyen GN, Rothstein SJ, Spangenberg G, Kant S. Role of microRNAs involved in plant response to nitrogen and phosphorous limiting conditions. Front Plant Sci. 2015;6 https://doi.org/10.3389/fpls.2015.00629.

42. Hsieh LC, Lin SI, Shih ACC, Chen JW, Lin WY, Tseng CY, Li WH, Chiou TJ. Uncovering small RNA-mediated responses to phosphate deficiency in arabidopsis by deep sequencing. Plant Physiol. 2009;151(4):2120-32. https:// doi.org/10.1104/pp.109.147280.

43. Liang $\mathrm{G}, \mathrm{Ai} \mathrm{Q}, \mathrm{Yu} \mathrm{DQ}$. Uncovering miRNAs involved in crosstalk between nutrient deficiencies in Arabidopsis. Sci Rep. 2015;5(1) https://doi.org/10.103 8/srep11813.

44. Zhang ZL, Liao H, Lucas WJ. Molecular mechanisms underlying phosphate sensing, signaling, and adaptation in plants. J Integr Plant Biol. 2014;56(3): 192-220. https://doi.org/10.1111/jipb.12163.

45. Baker A, Ceasar SA, Palmer AJ, Paterson JB, Qi WJ, Muench SP, Baldwin SA Replace, reuse, recycle: improving the sustainable use of phosphorus by plants. J Exp Bot. 2015;66(12):3523-40. https://doi.org/10.1093/jxb/erv210.

46. Smith AP, Fontenot EB, Zahraeifard S, DiTusa SF. Molecular components that drive phosphorus-remobilisation during leaf senescence. Annual Plant Rev. 2015:48:159-86.

47. Stigter KA, Plaxton WC. Molecular mechanisms of phosphorus metabolism and transport during leaf senescence. Plants. 2015;4(4):773-98. https://doi. org/10.3390/plants4040773.

48. Aerts R. Nutrient resorption from senescing leaves of perennials: are there general patterns? J Ecol. 1996;84(4):597-608. https:/doi.org/10.2307/2261481.

49. Bouain N, Shahzad Z, Rouached A, Khan GA, Berthomieu P, Abdelly C, Poirier $Y$, Rouached $H$. Phosphate and zinc transport and signalling in plants: toward a better understanding of their homeostasis interaction. J Exp Bot. 2014;65(20):5725-41. https://doi.org/10.1093/jxb/eru314. 
50. Nagarajan VK, Jain A, Poling MD, Lewis AJ, Raghothama KG, Smith AP. Arabidopsis Pht1;5 mobilizes phosphate between source and sink organs and influences the interaction between phosphate homeostasis and ethylene signaling. Plant Physiol. 2011;156(3):1149-63. https://doi.org/10.11 04/pp.111.174805.

51. Nussaume L, Kanno S, Javot H, Marin E, Pochon N, Ayadi A, et al. Phosphate import in plants: focus on the PHT1 transporters. Front Plant Sci. 2011;2 https://doi.org/10.3389/fpls.2011.00083.

52. Hassler S, Jung B, Lemke L, Novak O, Strnad M, Martinoia E, et al. Function of the golgi-located phosphate transporter PHT4;6 is critical for senescenceassociated processes in Arabidopsis. J Exp Bot. 2016;67(15):4671-84. https:// doi.org/10.1093/jxb/erw249.

53. Fahlgren N, Howell MD, Kasschau KD, Chapman EJ, Sullivan CM, Cumbie JS, Givan SA, Law TF, Grant SR, Dangl JL, Carrington JC. High-throughput sequencing of Arabidopsis microRNAs: evidence for frequent birth and death of miRNA genes. PLoS One. 2007;2(2):e219. https://doi.org/10.1371/ journal.pone.0000219.

54. Liu J, Yang L, Luan M, Wang Y, Zhang C, Zhang B, Shi J, Zhao FG, Lan W, Luan S. A vacuolar phosphate transporter essential for phosphate homeostasis in Arabidopsis. Proc Natl Acad of Sci USA. 2015;112(47):E6571E8. https://doi.org/10.1073/pnas.1514598112.

55. Liu TY, Huang TK, Yang SY, Hong YT, Huang SM, Wang FN, et al. Identification of plant vacuolar transporters mediating phosphate storage. Nature Comm. 2016;7:11095. https://doi.org/10.1038/ncomms11095.

56. Dai $X$, Zhao PX. psRNATarget: a plant small RNA target analysis server. Nucleic Acids Res. 2011;39(suppl_2):W155-W9.

57. Sun X, Xie ZQ, Zhang C, Mu Q, Wu WM, Wang BJ, Fang J. A characterization of grapevine of GRAS domain transcription factor gene family. Funct Integr Genomic. 2016;16(4):347-63. https://doi.org/10.1007/s10142-016-0479-y.

58. Perez-Amador MA, Abler ML, De Rocher EJ, Thompson DM, van Hoof A, LeBrasseur ND, et al. Identification of BFN1, a bifunctional nuclease induced during leaf and stem senescence in Arabidopsis. Plant Physiol. 2000;122(1): 169-79. https://doi.org/10.1104/pp.122.1.169.

59. Pontier D, Gan SS, Amasino RM, Roby D, Lam E. Markers for hypersensitive response and senescence show distinct patterns of expression. Plant Mol Biol. 1999;39(6):1243-55. https://doi.org/10.1023/A:1006133311402.

60. Waese J, Fan J, Pasha A, Yu H, Fucile G, Shi R, Cumming M, Kelley LA, Sternberg MJ, Krishnakumar V, Ferlanti E, Miller J, Town C, Stuerzlinger W, Provart NJ. ePlant: visualizing and exploring multiple levels of data for hypothesis generation in plant biology. Plant Cell. 2017;29(8):1806-21. https://doi.org/10.1105/tpc.17.00073.

61. Kaundal R, Saini R, Zhao PX. Combining machine learning and homologybased approaches to accurately predict subcellular localization in Arabidopsis. Plant Physiol. 2010;154(1):36-54. https://doi.org/10.1104/pp.11 0.156851 .

62. Lopez-Bucio J, Hernandez-Abreu E, Sanchez-Calderon L, Nieto-Jacobo MF, Simpson J, Herrera-Estrella L. Phosphate availability alters architecture and causes changes in hormone sensitivity in the Arabidopsis root system. Plant Physiol. 2002;129(1):244-56. https://doi.org/10.1104/pp.010934.

63. Lopez-Bucio J, Cruz-Ramirez A, Herrera-Estrella L. The role of nutrient availability in regulating root architecture. Curr Opin Plant Biol. 2003;6(3): 280-7. https://doi.org/10.1016/S1369-5266(03)00035-9.

64. Gu MA, Chen AQ, Sun SB, Xu GH. Complex regulation of plant phosphate transporters and the gap between molecular mechanisms and practical application: what is missing? Molec Plant. 2016;9(3):396-416. https://doi. org/10.1016/j.molp.2015.12.012.

65. Wege S, Khan GA, Jung JY, Vogiatzaki E, Pradervand S, Aller I, Meyer AJ, Poirier $Y$. The EXS domain of $\mathrm{PHO} 1$ participates in the response of shoots to phosphate deficiency via a root-to-shoot signal. Plant Physiol. 2016;170(1): 385-400. https://doi.org/10.1104/pp.15.00975.

66. Mudge SR, Rae AL, Diatloff E, Smith FW. Expression analysis suggests novel roles for members of the PHT1 family of phosphate transporters in Arabidopsis. Plant J. 2002;31(3):341-53. https://doi.org/10.1046/j.1365-313X.2 002.01356.x.

67. Ma C, Burd S, Lers A. miR408 is involved in abiotic stress responses in Arabidopsis. Plant J. 2015;84(1):169-87. https://doi.org/10.1111/tpj.12999.

68. Pant BD, Musialak-Lange M, Nuc P, May P, Buhtz A, Kehr J, Walther D, Scheible WR. Identification of nutrient-responsive arabidopsis and rapeseed microRNAs by comprehensive real-time polymerase chain reaction profiling and small RNA sequencing. Plant Physiol. 2009;150(3):1541-55. https://doi. org/10.1104/pp.109.139139.
69. Lin S-I, Santi C, Jobet E, Lacut E, El Kholti N, Karlowski WM, et al. Complex regulation of two target genes encoding SPX-MFS proteins by rice miR827 in response to phosphate starvation. Plant Cell Physiol. 2010;51(12):2119-31. https://doi.org/10.1093/pcp/pcq170.

70. Chevalier F, Perazza D, Laporte F, Le Henaff G, Hornitschek P, Bonneville JM, et al. GeBP and GeBP-like proteins are noncanonical leucine-zipper transcription factors that regulate cytokinin response in Arabidopsis. Plant Physiol. 2008;146(3):1142-54. https://doi.org/10.1104/pp.107.110270.

71. Curaba J, Herzog M, Vachon G. GeBP, the first member of a new gene family in Arabidopsis, encodes a nuclear protein with DNA-binding activity and is regulated by KNAT1. Plant J. 2003;33(2):305-17. https://doi.org/10.104 6/j.1365-313X.2003.01622.x.

72. Khare D, Mitsuda N, Lee S, Song WY, Hwang D, Ohme-Takagi M, Martinoia E, Lee Y, Hwang JU. Root avoidance of toxic metals requires the GeBP-LIKE 4 transcription factor in Arabidopsis thaliana. New Phytol. 2017;213(3):125773. https://doi.org/10.1111/nph.14242.

73. Perazza D, Laporte F, Balague C, Chevalier F, Remo S, Bourge M, et al. GeBP/ GPL transcription factors regulate a subset of CPR5-dependent processes. Plant Physiol. 2011;157(3):1232-42. https://doi.org/10.1104/pp.111.179804.

74. Farage-Barhom S, Burd S, Sonego L, Mett A, Belausov E, Gidoni D, Lers A. Localization of the Arabidopsis senescence- and cell death-associated BFN1 nuclease: from the ER to fragmented nuclei. Mol Plant. 2011;4(6):1062-73. https://doi.org/10.1093/mp/ssr045.

75. Farage-Barhom S, Burd S, Sonego L, Perl-Treves R, Lers A. Expression analysis of the BFN1 nuclease gene promoter during senescence, abscission, and programmed cell death-related processes. J Exp Bot. 2008;59(12):3247-58. https://doi.org/10.1093/jxb/ern176.

76. James M, Masclaux-Daubresse C, Marmagne A, Azzopardi M, Laine P, Goux $D$, et al. A new role for SAG12 cysteine protease in roots of Arabidopsis thaliana. Front Plant Sci. 2019;9 https://doi.org/10.3389/fpls.2018.01998.

77. Alves L, Niemeier S, Hauenschild A, Rehmsmeier M, Merkle T. Comprehensive prediction of novel microRNA targets in Arabidopsis thaliana. Nucleic Acids Res. 2009;37(12):4010-21. https://doi.org/10.1093/nar/ gkp272.

78. Allen E, Xie ZX, Gustafson AM, Carrington JC. microRNA-directed phasing during trans-acting siRNA biogenesis in plants. Cell. 2005;121(2):207-21. https://doi.org/10.1016/j.cell.2005.04.004.

79. Lauter N, Kampani A, Carlson S, Goebel M, Moose SP. MicroRNA172 down-regulates glossy15 to promote vegetative phase change in maize. Proc Natl Acad Sci U S A. 2005;102(26):9412-7. https://doi.org/10.1073/ pnas.0503927102.

80. Peng MS, Hannam C, Gu HL, Bi YM, Rothstein SJ. A mutation in NLA, which encodes a RING-type ubiquitin ligase, disrupts the adaptability of Arabidopsis to nitrogen limitation. Plant J. 2007:50(2):320-37. https://doi. org/10.1111/j.1365-313X.2007.03050.X.

81. Park BS, Yao T, Seo JS, Wong ECC, Mitsuda N, Huang CH, Chua NH. Arabidopsis nitrogen limitation adaptation regulates ORE1 homeostasis during senescence induced by nitrogen deficiency. Nat Plants. 2018:4(11): 898-903. https://doi.org/10.1038/s41477-018-0269-8.

82. Matallana-Ramirez LP, Rauf M, Farage-Barhom S, Dortay H, Xue GP, DrogeLaser W, et al. NAC transcription factor ORE1 and senescence-induced bifunctional nuclease1 (BFN1) constitute a regulatory cascade in Arabidopsis. Mol Plant. 2013;6(5):1438-52. https://doi.org/10.1093/mp/sst012.

83. Oh SA, Park JH, Lee Gl, Paek KH, Park SK, Nam HG. Identification of three genetic loci controlling leaf senescence in Arabidopsis thaliana. Plant J. 1997;12(3):527-35. https://doi.org/10.1111/j.0960-7412.1997.00527.x.

84. Hammond JP, Bennett MJ, Bowen HC, Broadley MR, Eastwood DC, May ST, Rahn C, Swarup R, Woolaway KE, White PJ. Changes in gene expression in Arabidopsis shoots during phosphate starvation and the potential for developing smart plants. Plant Physiol. 2003;132(2):578-96. https://doi.org/1 0.1104/pp.103.020941.

85. Linkohr Bl, Williamson LC, Fitter AH, Leyser HMO. Nitrate and phosphate availability and distribution have different effects on root system architecture of Arabidopsis. Plant J. 2002;29(6):751-60. https://doi.org/10.104 6/j.1365-313X.2002.01251.X.

86. Peret B, Desnos T, Jost R, Kanno S, Berkowitz O, Nussaume L. Root architecture responses: in search of phosphate. Plant Physiol. 2014;166(4): 1713-23. https://doi.org/10.1104/pp.114.244541.

87. Chien PS, Chiang CB, Wang ZR, Chiou TJ. MicroRNA-mediated signaling and regulation of nutrient transport and utilization. Curr Opin Plant Biol. 2017;39: 73-9. https://doi.org/10.1016/j.pbi.2017.06.007. 
88. Park BS, Seo JS, Chua NH. NITROGEN LIMITATION ADAPTATION recruits PHOSPHATE2 to target the phosphate transporter PT2 for degradation during the regulation of Arabidopsis phosphate homeostasis. Plant Cell. 2014;26(1):454-64. https://doi.org/10.1105/tpc.113.120311.

89. Wang DLY, Lv SL, Jiang P, Li YX. Roles, regulation, and agricultural application of plant phosphate transporters. Front Plant Sci. 2017;8:817. https://doi.org/10.3389/fpls.2017.00817.

90. Devaiah BN, Madhuvanthi R, Karthikeyan AS, Raghothama KG. Phosphate starvation responses and gibberellic acid biosynthesis are regulated by the MYB62 transcription factor in Arabidopsis. Mol Plant. 2009;2(1):43-58. https://doi.org/10.1093/mp/ssn081.

91. Yan J, Gu Y, Jia X, Kang W, Pan S, Tang X, Chen X, Tang G. Effective small RNA destruction by the expression of a short tandem target mimic in Arabidopsis. Plant Cell. 2012;24(2):415-27. https://doi.org/10.1105/tpc.111. 094144.

92. Coutu C, Brandle J, Brown D, Brown K, Miki B, Simmonds J, Hegedus DD PORE: a modular binary vector series suited for both monocot and dicot plant transformation. Transgenic Res. 2007;16(6):771-81. https://doi.org/10.1 007/s11248-007-9066-2.

93. Clough SJ, Bent AF. Floral dip: a simplified method for Agrobacteriummediated transformation of Arabidopsis thaliana. Plant J. 1998;16(6):735-43. https://doi.org/10.1046/j.1365-313x.1998.00343.x.

94. Porra RJ, Thompson WA, Kriedemann PE. Determination of accurate extinction coefficients and simultaneous-equations for assaying chlorophylla and chlorophyll-b extracted with 4 different solvents - verification of the concentration of chlorophyll standards by atomic-absorption spectroscopy. Biochim Biophys Acta. 1989;975(3):384-94. https://doi.org/10.1016/S0005-2 728(89)80347-0.

95. Chen CF, Ridzon DA, Broomer AJ, Zhou ZH, Lee DH, Nguyen JT, Barbisin M, Xu NL, Mahuvakar VR, Andersen MR, Lao KQ, Livak KJ, Guegler KJ. Real-time quantification of microRNAs by stem-loop RT-PCR. Nucleic Acids Res. 2005. 33(20):e179. https://doi.org/10.1093/nar/gni178.

96. Pant BD, Buhtz A, Kehr J, Scheible WR. MicroRNA399 is a long-distance signal for the regulation of plant phosphate homeostasis. Plant J. 2008;53(5):731-8. https://doi.org/10.1111/j.1365-313X.2007.03363.x.

97. Ma C, Lu Y, Bai SL, Zhang WN, Duan XW, Meng D, Wang Z, Wang A, Zhou Z, Li T. Cloning and characterization of miRNAs and their targets, including a novel miRNA-targeted NBS-LRR protein class gene in apple (Golden Delicious). Mol Plant. 2014;7(1):218-30. https://doi.org/10.1093/mp/sst101.

98. Ames BN. Assay of inorganic phosphate, total phosphate and phosphatases. Methods Enzymol. 1966:8:115-8.

99. Hurley BA, Tran HT, Marty NJ, Park J, Snedden WA, Mullen RT, Plaxton WC. The dual-targeted purple acid phosphatase isozyme AtPAP26 is essential for efficient acclimation of Arabidopsis to nutritional phosphate deprivation. Plant Physiol. 2010;153(3):1112-22.

\section{Publisher's Note}

Springer Nature remains neutral with regard to jurisdictional claims in published maps and institutional affiliations.

Ready to submit your research? Choose BMC and benefit from:

- fast, convenient online submission

- thorough peer review by experienced researchers in your field

- rapid publication on acceptance

- support for research data, including large and complex data types

- gold Open Access which fosters wider collaboration and increased citations

- maximum visibility for your research: over $100 \mathrm{M}$ website views per year

At $\mathrm{BMC}$, research is always in progress.

Learn more biomedcentral.com/submissions 